\title{
Elevated METTL16 expression in PDAC confers synthetic lethality to PARP inhibition by antagonizing MRE11-mediated DNA end resection
}

\section{Xiangyu Zeng}

Department of Oncology, Mayo Clinic

Fei Zhao

Mayo Clinic

Jake Kloeber

Mayo Clinic https://orcid.org/0000-0002-6646-564X

Rajashree Deshpande

The University of Texas at Austin

\section{Georges Mer}

Mayo Clinic https://orcid.org/0000-0002-1900-1578

\section{Tanya Paull}

The University of Texas at Austin

\section{Zhenkun Lou ( $\boldsymbol{\sim}$ Lou.zhenkun@mayo.edu )}

Department of Oncology, Mayo Clinic https://orcid.org/0000-0003-1938-3091

\section{Article}

Keywords: pancreatic ductal adenocarcinoma (PDAC), METTL16, treatment strategies

Posted Date: June 25th, 2021

DOl: https://doi.org/10.21203/rs.3.rs-598847/v1

License: (9) This work is licensed under a Creative Commons Attribution 4.0 International License. Read Full License

Version of Record: A version of this preprint was published at Nature Cancer on September 22nd, 2022. See the published version at https://doi.org/10.1038/s43018-022-00429-3. 


\section{Abstract}

Pancreatic ductal adenocarcinoma (PDAC) is one of the most lethal cancers worldwide. Characterization of the recurrent genetic alterations in PDAC will yield improved understanding and therapies for this disease. Here, we report that PDAC patients with elevated expression of METTL16, one of the "writers" of RNA $\mathrm{N}^{6}$-methyladenosine $\left(\mathrm{m}^{6} \mathrm{~A}\right)$ modification, may benefits from poly (ADP-ribose) polymerase (PARP) inhibitor treatment. Mechanistically, METTL16 interacts with MRE11 in an RNA-dependent manner; and, this interaction inhibits MRE11's exonuclease activity in a methyltransferase-independent manner, thereby repressing DNA end resection. Upon DNA damage, ATM phosphorylates METTL16 at Ser419 within its C terminus, resulting in METTL16 conformational change and autoinhibition of its RNA binding. This dissociates the METTL16-RNA-MRE11 complex and releases inhibition of MRE11. Concordantly, PDAC cells with high METTL16 expression levels show increased sensitivity to PARP inhibitors, especially when combined with gemcitabine. Thus, our findings have revealed a role for METTL16 in homologous recombination repair and suggest that combination of PARP inhibitors with gemcitabine could be an effective treatment strategy for PDAC patients with high METTL16 expression.

\section{Introduction}

Pancreatic ductal adenocarcinoma (PDAC) is one of the most aggressive malignancies with fewer than $10 \%$ of patients surviving 5 years ${ }^{1-3}$. Gemcitabine is the most widely used single agent chemotherapeutic treatment for locally advanced and metastatic PDAC ${ }^{4,5}$. However, the efficacy of gemcitabine remains modest. Currently emerging clinical studies are underway to explore combination treatment benefits of gemcitabine with other targeted agents for novel and more effective therapeutic strategies for PDAC ${ }^{6,7}$. Poly (ADP-ribose) polymerase (PARP) is a nuclear enzyme that plays a key role in DNA repair ${ }^{8}$. PARP inhibitors (PARPi) are the first clinically approved drugs designed to exploit synthetic lethality ${ }^{9-11}$. Tumors harboring germline or somatic BRCA1/2 mutations are sensitive to PARPi because of an underlying homologous recombination (HR) defect ${ }^{12,13}$. PARPi also show promising activity in more common cancers that share defective HR. Indeed, besides breast and ovarian cancer, PARP inhibitors are now an FDA approved standard treatment option as maintenance therapy for metastatic PDAC patients who harbor pathogenic germline BRCA1/2 mutations ${ }^{14}$. However, only about $10 \%$ of PDAC patients harbor pathogenic mutations of these HR genes, leaving most patients missing out on the encouraging treatment strategy ${ }^{11,15,16}$.

DNA double-strand breaks (DSBs) represent the most cytotoxic DNA lesions that can compromise genomic integrity and viability ${ }^{17}$. To repair DSBs, eukaryotic cells employ two major strategies, nonhomologous end-joining (NHEJ) and homologous recombination (HR $)^{18,19}$. Error-prone NHEJ directly joins the broken ends together and may create mutations ${ }^{20}$. On the contrary, HR is a high-fidelity and error-free DNA repair mechanism that occurs predominantly during the $S$ and $\mathrm{G} 2$ phases of the cell cycle, where a homologous template is available for repair ${ }^{21,22}$. The MRE11/RAD50/NBS1 (MRN) complex acts as a sensor of DSBs and is quickly recruited to the damage sites ${ }^{23}$. When DSBs occur, MRE11 can initiate 
DNA end resection to generate short stretches of single-stranded DNA (SSDNA), which is a critical step in $\mathrm{HR}^{24}$. Subsequently, EX01/DNA2 are recruited to DSBs sites to expand end resection. Therefore, MRE11 is the functional subunit of the MRN complex, which plays a key role in DNA repair regulation. Meanwhile, the function of MRE11 is strictly regulated in cells ${ }^{25,26}$. PLK1 phosphorylates MRE11 at Ser649 to inhibit its DNA binding activity and antagonize HR repair ${ }^{27}$. UBQLN4 promotes the ubiquitination and degradation of chromatin MRE11, thus affects DSBs repair choice. In addition to post translational modification, protein-protein interactions were also reported to affect MRE11 function ${ }^{28}$. C1QBP interacts with the GAR domain of MRE11 and inhibits the nuclease activity of MRE1 ${ }^{29}$. DYNLL1 and Nej1 were also recently identified as MRE11 interacting proteins and reported to inhibit MRE11-mediated end resection ${ }^{30}$.

N6-methyladenosine $\left(m^{6} \mathrm{~A}\right)$ methylation is one of the most common RNA modifications ${ }^{31,32} \cdot \mathrm{m}^{6} \mathrm{~A}$ modifications occur via the $\mathrm{m}^{6} \mathrm{~A}$ methyltransferases called "writers", they are removed by demethylases termed "erasers", and they are recognized by $\mathrm{m}^{6} \mathrm{~A}$-binding proteins called "readers". Methyltransferaselike family members of METTL3, METTL14, and METTL16 have been shown acting as "writers" to initiate the $\mathrm{m}^{6} \mathrm{~A}$ modification process ${ }^{33}$. Recently, Xiang et al. reported that $\mathrm{m}^{6} \mathrm{~A}$ modification can help to recruit DNA polymerase $\mathrm{k}$ ( $\mathrm{Pol} \mathrm{k}$ ) for nucleotide excision and transl-esion synthesis-mediated single-strand breaks in response to ultra-violet (UV) irradiation ${ }^{34}$. Furthermore, Zhang et al. revealed that METTL3 modulates accumulation of DNA-RNA hybrids at DSBs sites depending on its methyltransferase (MTase) activity, and then promote HR repair by assisting recruitment of RAD51 and BRCA $1^{35}$. In this study, we found that METTL16-RNA complexes are a natural and potent inhibitor of MRE11-mediated DNA end resection. This regulation is independent of the MTase activity of METTL16. More importantly, we found that PDAC tumor cells with high METTL16 expression can be selectively killed by PARP inhibitors, especially when combined with gemcitabine. Therefore, our findings reveal a novel DNA end resection regulatory mechanism of METTL16-RNA complex and provide a strategy for the treatment of PDAC with immediate clinical applicability.

\section{Results}

\section{METTL16 expression is directly correlated with accumulated DSBs and increased overall survival in PDAC patients}

To address the clinical relevance of these three core RNA methyltransferases function in $\mathrm{m}^{6} \mathrm{~A}$ modification, METTL3, METTL14 and METTL16 in PDAC, we performed immunohistochemical staining in a PDAC tissue microarray containing a cohort of pancreatic normal and carcinoma samples (Fig. 1a and Extended Data Fig. 1a). We first noticed that METTL14 and METTL16, but not METTL3, were highly expressed in PDAC carcinoma tissues compared to normal tissue (Extended Data Fig. 1b). In addition, analysis of date from The Cancer Genome Atlas (TCGA) revealed that METTL14 and METTL16 mRNA levels are also markedly upregulated in PDAC carcinoma samples (Extended Data Fig. 1c). Importantly, Kaplan-Meier survival analysis from tissue microarray or TCGA data sets indicate that elevated 
expression of METTL3, METTL14, and METTL16 positively correlate with favorable survival in patients with PDAC (Fig. 1b and Extended Data Fig. 1d). Together, these findings point to a role for METTL3, METTL14 and METTL16 as biomarkers of better clinical outcome in PDAC.

We next asked why these three RNA methyltransferases are associated with PDAC patient survival. Since chemotherapy utilizing DNA damage-inducing agents, such as gemcitabine and platinum, is the standard treatment strategy for PDAC patients ${ }^{36-38}$, we assessed DSBs level in the tissue microarray using S139 phosphorylated histone $\mathrm{H} 2 \mathrm{AX}$ ( $\mathrm{g}-\mathrm{H} 2 \mathrm{AX}$ ) as a marker (Fig. 1c). We surprisingly found that only METTL16 high expression is related to elevated accumulation of DSBs (Fig. 1d). On the contrary, METTL3 high expression showed decreased DSBs, while METTL14 expression doesn't show significant relevance to DSBs accumulation (Fig. 1d). Moreover, we utilized a DR-GFP/EJ5-GFP reporter system to further evaluate the function of these three RNA $\mathrm{m}^{6} \mathrm{~A}$ methyltransferases in DSBs repair (Fig. 1e). The results suggest that METTL3 knockdown decreases both HR and NHEJ, which is consistent with previously published studies ${ }^{34,35}$; METTL14 knockdown had no significant effect on HR or NHEJ; and, METTL16 knockdown significantly increased HR, but had no apparent effects on NHEJ (Fig. $1 \mathrm{f}$ and Extended Data Fig. 1e). All the DSBs repair reporter data are consistent with the IHC results. Taken together, these results suggest that METTL16 blocks HR and high expression of METTL16 indicates better outcome with increased DSBs in PDAC.

\section{METTL16 regulates HR repair through ATM phosphorylation}

To further investigate the potential role of METTL16 in HR repair, we used two independent sgRNAs to knockout (KO) METTL16. We then used immunofluorescent to check g-H2AX foci induced by ionizing radiation (IR) in cells depleted of METTL16 and with re-expression METTL16. Data showed that although METTL16-deficiency did not alter the induction of g-H2AX foci, the percent of $\mathrm{g}-\mathrm{H} 2 \mathrm{AX}$ foci positive cells in METTL16 KO cells were significantly lower than WT cells 8h after IR treatment (Fig. 2a-c), suggesting enhanced DNA repair in METTL16-deficient cells. And this difference was abrogated by re-expression of METTL16 in METTL16 KO cells, confirming the effect of METTL16 is on target. Consistently, western blots examining g-H2AX kinetics after IR showed that g-H2AX levels were similar between WT and METTL16 KO cells at $1 \mathrm{~h}$ after DNA damage; whereas the g-H2AX levels in METTL16 KO cells were significantly lower than WT cells starting $4 \mathrm{~h}$ after IR treatment (Fig. 2d,e). In addition, expression of METTL16 could reverse the change in kinetics of g-H2AX accumulation caused by METTL16 KO (Fig. 2d,e). Furthermore, we measured the effect of METTL16 depletion on DNA damage using the neutral comet DNA repair assay. As shown in Fig. 2f,g, the tail moments of METTL16 KO cells were markedly shorter than those of WT cells at 4h after IR treatment, although no significant differences were observed at $0.5 \mathrm{~h}$ after IR treatment. Re-expression of METTL16 reversed this change. These results suggest that METTL16 play a negative role in DNA repair.

Analysis of METTL16 protein sequence showed that there are two potential ATM-phosphorylation consensus sequences at Ser419 and Ser455 (Fig. 2h). Thus, we speculated that METTL16 might be a substrate of ATM. Indeed, we found that METTL16 is phosphorylated at an ATM/ATR consensus motif in 
an ATM-dependent manner after IR (Fig. 2i). By mutating these putative ATM phosphorylation sites on METTL16 individually, we confirmed that Ser419 of METTL16 is phosphorylated by ATM following DNA damage (Fig. 2j). We next tested whether METTL16 phosphorylation affects its role in HR repair. In cells depleted of endogenous METTL16 and reconstituted with WT, S419A, or S419D METTL16, we found that WT, but not a phosphorylation mimicking mutant S419D could rescue HR repair efficiency. Furthermore, reconstitution with a phosphorylation defective mutant S419A further decreased HR repair efficiency (Fig. $2 \mathrm{k}$ ). These results indicate that ATM-mediated phosphorylation of METTL16 after DNA damage attenuates its inhibitory role in HR. To further address our hypothesis, we examined g-H2AX foci and expression kinetics in METTL16 depletion cells reconstitution with WT, S419A, or S419D METTL16 (Extended Data Fig. 2a) following IR treatment. Indeed, the results demonstrate that the S419A mutation caused more sustained g-H2AX foci and g- $\mathrm{H} 2 \mathrm{AX}$ chromatin fraction while the S419D mutant has an opposite effect (Extended Data Fig. 2b-e). Finally, we conducted neutral comet assays in METTL16depleted cells reconstituted with WT, S419A, or S419D METTL16. The tail moments could be restored by WT METTL16, but not the S419D mutant. Re-expressing S419A METTL16 further increased tail moments (Extended Data Fig. 2f,g). These data suggest that ATM-mediated phosphorylation of METTL16 decreases its inhibitory function in HR repair.

\section{METTL16 suppresses DNA end resection through interaction with MRE11}

Because our data suggested that METTL16 inhibits HR repair, we next studied the underlying mechanism. First, we tested BRCA1 and PALB2 recruitment to DSBs sites after IR treatment. The results showed no significant changes of BRCA1 or PALB2 foci in METTL16-depletion cells exposed to IR (Extended Data Fig. 3a-d). However, there was a notable increase of RPA32 and RAD51 foci in METTL16-depleted cells (Fig. 3a-d). This difference of RPA32 and RAD51 foci formation could be abrogated by re-expression of METTL16 in METTL16 KO cells (Fig. 3a-d). Increased RPA32 foci formation implies elevated ssDNA formation by DNA end resection in METTL16-depleted cells ${ }^{39}$. We confirmed this using a BrdU incorporation assay (Fig. 3e,f), which is commonly used to detect DNA end resection generated ssDNA ${ }^{40}$. BrdU signals were significantly increased in METTL $16 \mathrm{KO}$ cells and could be rescued with re-expression of METTL16, further supporting an inhibitory role for METTL16 in DNA end resection (Fig. 3e,f). Meanwhile, the lack of METTL16 did not affect BRCA1, PALB2, Rad51, or RPA32 expression (Extended Data Fig. 3e). In support of the IF results, analysis of chromatin-enriched fractions revealed that KO of METTL16 increases the accumulation of RPA32 and RAD51 (Fig. 3g), but not PALB2 and BRCA1 (Extended Data Fig. 3f) chromatin-enriched protein extracts after IR. Together, these results suggest that METTL16 suppresses HR-mediated DSBs repair through DNA end resection.

We have shown that METTL16 could be phosphorylated by ATM upon DNA damage, we next tested whether this phosphorylation also has a role in METTL16 mediated DNA end resection inhibition. We expressed WT, S419A or S419D METTL16 in METTL16-null cells and found that the WT and S419A mutant reversed the increase of RPA32 and BrdU foci caused by METTL16 depletion, but not the S419D mutant (Fig. 3h,i and Extended Data Fig. 3g,h). In agreement with this, enrichment of RPA32 on chromatin fractions could be reversed by the WT and S419A mutant METTL16, but not the S419D mutant (Fig. 3j). 
These results collectively suggest that METTL16 blocks DNA end resection which is relieved by ATMmediated phosphorylation at Ser419 after DNA damage.

In order to investigate the molecular mechanism by which METTL16 regulates DNA end resection, we tested the association of METTL16 with key DNA end resection factors involved in the initial steps of HR. We observed that METTL16 interacted with MRE11, RAD50, and NBS1 (MRN complex), and interestingly, these interactions were sharply attenuated after DNA damage (Fig. 3k-m). MRE11 is the core of the MRN complex that can initiate DNA end resection ${ }^{24,41}$, and we have demonstrated that METTL16 inhibits HR by attenuating DNA end resection. This implied that METTL16 functions in DNA end resection through regulating MRE11 activity. Of note, METTL16 depletion did not affect the expression of MRE11 (Extended Data Fig. 3i), or MRN complex stability (Extended Data Fig. 3j). Since the MRN complex also initiates DNA damage repair signaling through activation of the ATM kinase ${ }^{25}$, we next tested whether METTL16 depletion affects ATM activation and found that ATM activation is intact after depleting METTL16 (Extended Data Fig. 3k).

Based on our data that the interaction between METTL16 and MRE11 is decreased after DNA damage, we hypothesized that ATM-mediated phosphorylation of METTL16 after DNA damage influences its interaction with MRE11. Indeed, an ATM specific inhibitor (ATMi KU55933) abolished the DNA damage induced METTL16-MRE11 dissociation (Fig. 3N). Furthermore, the interaction between S419A mutant and MRE11 did not decrease after DNA damage (Fig. 3o). On the other hand, the S419D mutant could not interact with MRE11 (Extended Data Fig. 3I). Taken together, our data demonstrate that METTL16 interacts with MRE11 and suppresses DNA end resection. ATM-mediated METTL16 Ser419 phosphorylation after DNA damage attenuates this interaction and inhibition.

\section{RNA inactivates MRE11 through orchestrating the METTL16-RNA-MRE11 complex formation}

MRE11 possesses two DNA binding domains (DBD) at its $C$ terminus to bind DNA, while METTL16 contains four RNA binding domains (RBD) within its $N$ terminus to bind RNA ${ }^{42,43}$. Therefore, we sought to determine whether METTL16 directly interacts with MRE11 through a DNA or RNA dependent manner. The results showed that RNase rather than DNase abrogated the interaction between METTL16 and MRE11 (Fig. 4a), suggesting that METTL16 interacts with MRE11 in an RNA dependent manner. To investigate the region responsible for METTL16-MRE11 interaction, serial truncated mutants of MRE11 (Fig. 4b) and METTL16 (Fig. 4d) were generated. The MRE11 MD5 mutant (DNA binding domain 2 deletion) or the METTL16 MR2 mutant (RNA binding domain 2 deletion) were unable to participate in interactions between METTL16 and MRE11, respectively (Fig. 4c,e). These results suggest that the MRE11 DBD2 domain and METTL16 RBD2 domain are essential for the interaction between METTL16 and MRE11, which further supports our conclusion that METTL16 interacts with MRE11 via RNA.

METTL16 targets a distinct set of RNAs sharing a consensus sequence of UACAGAGAA for $\mathrm{m}^{6} \mathrm{~A}$ modification ${ }^{44,45}$. To further validate that METTL16 interacts with MRE11 through RNA, we synthesized biotin labeled U6 snRNA, a known METTL16 substrate, and a control RNA (replace UACAGAGAA with 
AAAAAAAAA) to perform RNA pull-down assays. The data showed that the METTL16 MR2 mutant greatly decreased RNA binding (Fig. 4f). Interestingly, the MRE11 MD5 mutant also showed decreased RNA binding (Fig. 4g).

Because the MRN complex possesses 3'-5' exonuclease activity in vivo and in vitro ${ }^{23,25}$, we next evaluated whether METTL16 also regulates the nuclease activity of MRE11. MRE11/RAD50/NBS1 complex was expressed and purified from insect cells, and METTL16 proteins were purified from HEK293T (Extended Data Fig. 4a). We confirmed that purified MRN complex efficiently catalyzed 3' to 5' exonucleolytic degradation of different substrates in vitro, including with blunt-end dsDNA and dsDNA with $3^{\prime}$ and 5' overhangs (Fig. 4h,i). RNA or METTL16 alone had little effect on the enzymatic activity of MRN (Extended Data Fig. 4b,c). However, the MRN exonucleolytic digestion of dsDNA substrates was significantly repressed by the METTL16-RNA complex (Fig. 4h,i). Collectively, these data demonstrate that METTL16-RNA complex suppresses MRE11 exonuclease activity. Furthermore, unlike WT METTL16, reconstitution with MR2 mutant METTL16 failed to reverse g-H2AX foci (Extended Data Fig. 4e,f), tail moments (Extended Data Fig. 4g,h), the increase of RPA32 and BrdU foci (Fig. 4j,k and Extended Data Fig. 4i,j), RPA32 accumulation in chromatin fractions (Fig. 4m) and HR repair efficiency (Fig. 4I) caused by METTL16 depletion, suggesting that RNA binding is required for METTL16-mediated DNA end resection and HR suppression.

METTL16 is a highly conserved methyltransferase with orthologs found in organisms from E.coli to human ${ }^{44}$. It induces $\mathrm{m}^{6} \mathrm{~A}$ modification and plays an important role in RNA stability and splicing ${ }^{44}$. We next asked whether the effect of METTL16 is dependent on its enzymatic activity. We observed that the catalytically inactive F187G mutant METTL16 was still able to interact with MRE11 and this interaction was attenuated after DNA damage (Fig. 4n), suggesting that the MTase activity of METTL16 does not affect its interaction with MRE11. In cells depleted of endogenous METTL16 and reconstituted with WT or F187G mutant METTL16, both WT and F187G mutant METTL16 had similar effect on HR repair efficiency (Fig. 40), g-H2AX foci formation (Extended Data Fig. 4l,m), tail moments (Extended Data Fig. $4 n, 0$ ), the increase of RPA32 and BrdU foci (Fig. 4p,q and Extended Data $4 p, q$ ), and RPA32 accumulation in chromatin fractions (Fig. 4r).

Taken together, these data suggest that the interaction of METTL16-RNA-MRE11, but not the MTase activity of METTL16, is important for the inhibition of MRE11 nuclease activity (Fig. 4s).

\section{DNA damage induces METTL16 conformational change and affects its interaction with MRE11}

Because METTL16-mediated MRE11 inhibition requires its RNA binding, and ATM phosphorylation could relieve this inhibition, we next tested whether ATM-mediated phosphorylation of METTL16 Ser419 affects its RNA binding capacity. We performed an RNA pull-down assay using U6 snRNA, which has been shown to bind METTL16, and found that the interaction between METTL16 WT, but not the S419A mutant, with U6 snRNA significantly decreased upon DNA damage (Fig. 5a). In addition, the S419D mutant METTL16 
could not interact with U6 snRNA (Fig. 5b). These results suggest that ATM phosphorylation of METTL16 decreases its RNA binding and subsequent MRE11 inhibition.

As METTL16 phosphorylation at Ser419 showed decreased affinity to RNA and MRE11, we speculated that ATM-mediated Ser419 phosphorylation could induce a conformational change in METTL16 through a phosphorylation-mediated autoinhibition mechanism. To test this hypothesis, molecular dynamics (MD) simulations were performed to simulate the possible interaction between a METTL16 C-terminal peptide fragment (residues 413-425), phosphorylated at Ser419 (pS419) or unmodified, with METTL16 Nterminal RNA-binding domain. After a few nanoseconds of simulation, the METTL16 phosphopeptide, but not the unmodified peptide, adopted a stable conformation within the RNA binding pocket of METTL16 (Fig. 5c,d). After only $100 \mathrm{~ns}$ of simulation, the unmodified peptide had already moved away from its initial site and kept diffusing away from the $\mathrm{N}$-terminal domain during the course of the simulation (Extended Data Fig. 5a). Analysis of the $1 \mathrm{~s}$ simulation of METTL16 phosphopeptide suggested a tight interaction with an average of $12.45 \pm 2.44$ intermolecular hydrogen bonds $(20.77 \pm 3.88$ intermolecular polar pairs) (Fig. 5e). On the contrary, there was only an average of $3.17 \pm 2.58$ intermolecular hydrogen bonds (5.42 \pm 4.29 intermolecular polar pairs) formed between the native peptide and the METTL16 Nterminal domain (Fig. 5f). The root mean square (RMS) fluctuation analysis indicated that residues 416423 were more rigid than the other peptide residues (Extended Data Fig. 5b), with pS419 being the most rigid residue because of the intermolecular hydrogen bonds established with the phosphate group (Fig. $5 \mathrm{~g}$ ). However, the RMS fluctuations of each residue in the unmodified Ser419 peptide were much larger than the phosphopeptide (Extended Data Fig. 5c). The 3D structure of the phosphopeptide in complex with METTL16 N-terminal domain was extracted from the MD trajectory at the end of the simulation and was compared to the structure of the same domain in complex with an RNA molecule (PDB entry 6DU4). Interestingly, we observed that the METTL16 N-terminal domain RNA binding loop (residues 187-221) changed conformation when the domain bound the phosphopeptide (hot pink) in comparison to when it bound RNA (blue) (Fig. 5h and Extended Data Fig. 5d), but not when involved in the interaction with the non-phosphopeptide (Extended Data Fig. 5d,e). The phosphopeptide specifically interacted with the key RNA-binding Arg82 and Arg282 residues of METTL16 (Fig. 5g). Because of the overlapping binding sites within the METTL16 N-terminal domain for RNA and the METTL16 C-terminal segment harboring pS419, it is reasonable to suggest that phosphorylation of Ser419 displaces RNA from METTL16. In other words, METTL16 phosphorylated at Ser419 would be inhibitory for RNA binding through a conformational change.

To further validate the conformational change of METTL16 after DNA damage, we constructed METTL16 $\mathrm{N}$ terminus (1-300 aa) and $\mathrm{C}$ terminus (301-562 aa) expression constructs (Fig. 5i). We first noticed that METTL16 C terminus can still be phosphorylated by ATM following IR treatment (Extended Data Fig. 5f). Moreover, we observed that METTL16 with a WT C terminus, but not the $C$ terminus with the S419A mutation, strongly bound to the METTL16 N terminus after DNA damage (Fig. 5j). Furthermore, METTL16 $\mathrm{C}$ terminus with the S419D mutation was able to bind stronger with the METTL16 $\mathrm{N}$ terminus (Extended Data Fig. 5g). We next asked whether the METTL16 $\mathrm{C}$ terminus harboring the S419D mutation could compete with the binding between RNA and METTL16. The results indicate that the METTL16 C terminus 
domain with the S419D mutant completely disrupted the interaction between full-length METTL16 and U6 snRNA (Fig. 5k).

Next, we performed duo-link in situ assays to confirm the interaction between the $\mathrm{N}$ terminus and $\mathrm{C}$ terminus of METTL16 (Fig. 5l and Extended Data Fig. 5h). The result showed that for METTL16 WT, but not for the S419A mutant, the $\mathrm{C}$ terminal region was in close proximity to the $\mathrm{N}$ terminal region after DNA damage (Fig. 5m,n). In addition, for the METTL16 S419D mutant, the $C$ terminus shows a close proximity to the $\mathrm{N}$ terminal region in the absence of DNA damage, in contrast to WT METTL16 (Extended Data Fig $5 \mathrm{i}, \mathrm{j})$. To address the possibility that METTL16 may function as an oligomer after DNA damage, we tested METTL16 blots with native-polyacrylamide gel and only detected one band of METTL16 before or after DNA damage (Extended Data Fig. 5k), suggesting that METTL16 functions in cells as a monomer. Taken together, our results suggest that METTL16 undergoes a conformational change after DNA damage, and ATM mediated Ser419 phosphorylation promotes the binding of $\mathrm{C}$ terminus and $\mathrm{N}$ terminus which abrogates the RNA binding activity.

To further validate whether the two key RNA binding residues R82 and R282, which are predicted to form hydrogen bonds with pS419, play a key role in phosphorylation mediated METTL16 conformational change, we constructed METTL16 R82E/R282E (2RE) mutant by switching the positive charge residue arginine to negative charge residue glutamate. The result showed a significantly decreased interaction between the $\mathrm{N}$ terminus and $\mathrm{C}$ terminus in the 2RE mutant upon DNA damage (Fig. 50 and Extended Data Fig. 5l). In addition, proximity ligation assays also showed that the 2RE mutants abolished the duo-link signals induced by DNA damage (Fig. 5p,q), suggesting that the two arginine residues within the RNA binding domain are important for the docking of C-terminal fragment.

Collectively, these results demonstrated that DNA damage induces a METTL16 conformational change, resulting in its dissociation from RNA (Fig. 5r). The two positively charged arginine residues (R82 and R282) play a significant role in binding the phosphorylated C-terminal region of METTL16 to promote this conformational change.

\section{METTL16 Expression Sensitizes Tumor Cells to PARP1/2 Inhibition}

PARP inhibition was initially applied as a synthetic lethal therapy for breast and ovarian cancer patients with $B R C A 1 / 2$-mutant ${ }^{12,13}$. Subsequently, PARP inhibitors were found to work efficiently in tumors with defects in HR repair caused by mechanisms other than $B R C A$ mutations ${ }^{7,38}$. Since we found that a subset of PDAC has high METTL16 expression and that METTL16 expression leads to HR deficiency, we sought to explore the therapeutic potential of PARPi in PDAC. In PDAC cell lines harboring WT BRCA1/2, those with high expression of METTL16 were more sensitive to olaparib treatment by MTS assay compared to those with low expression of METTL16 (Fig. 6a,b). Next, we depleted endogenous METTL16 in the METTL16-high cell lines SW1990 (Fig. 6c) and PANC-1 (Extended Data Fig. 6a) using METTL16-specific sgRNAs and restored with METTL16 WT. The 2-D colony formation assays exhibited that control and METTL16 re-expressing cells were very sensitive to olaparib treatment; however, knockout of METTL16 
resulted in decreased sensitivity to olaparib (Fig. 6d,e and Extended Data Fig. 6b,c). Similar results were obtained from soft agar colony formation assays (Fig. 6f,g and Extended Data Fig. 6d,e). We next asked whether olaparib can work efficiently in vivo. We utilized xenograft tumor models inoculated with SW1990 METTL16 KO cells or SW1990 METTL16 KO cells re-expressing METTL16 (Extended Data Fig. 6f) and found that olaparib treatment markedly inhibited the growth of METTL16-positive SW1990 tumors in mice compared to those lacking METTL16 (Extended Data Fig. 6g,i). Meanwhile, the mice body weight showed similar growth rates in these four groups (Extended Data Fig. 6j). Furthermore, we collected three fresh PDAC tumors (PDAC1-3) with varying levels of METTL16 expression (Fig. 6h-j). We successfully established patient-derived xenograft (PDX) models from PDAC-2 and PDAC-3 (Fig. 6h). The PDX tumors derived from PDAC-2, which had higher METTL16 expression than PDAC-3, showed significant greater sensitivity to olaparib than those from PDAC-3 (Fig. 6k-m). Besides, the mice body weight in treat or untreated groups exhibited no significant difference (Fig. 6n). Together, these results support the hypothesis that high METTL16 expression is synthetic lethal with PARPi in PDAC cells.

\section{PARPi and gemcitabine show synergy in killing tumor cells expression METTL16}

Gemcitabine is the most extensively used single agent chemotherapeutic strategy for locally advanced and metastatic PDAC ${ }^{15,16}$. Patients with defective homologous DNA repair pathways are susceptible to chemotherapy and PARP inhibitor therapy ${ }^{10,11,46}$. Indeed, based on the Phase III POLO trial, PARP inhibitors are now an FDA approved standard treatment option as maintenance therapy for metastatic PDAC patients who harbor pathogenic germline BRCA1/2 mutations. However, only about $10 \%$ of PDAC patients harbor pathogenic mutations of these HR genes ${ }^{5,47}$, leaving most patients with fewer treatment options. Additionally, there may be greater prominent advantage when PARP inhibitors and gemcitabine are combined. Knowing that METTL16 expression induces HRD, we postulated that PARP inhibitors might facilitate PDAC tumor cell sensitivity to gemcitabine. Indeed, 2-D and soft agar colony formation assays presented that olaparib synergized with gemcitabine in SW1990 and PANC-1 METTL16 KO cells reexpressing METTL16 (Fig. 7a-d and Extended Data Fig. 7a-d). Furthermore, we examined the in vivo combination efficacy of olaparib and gemcitabine in xenografts and PDX (PDAC-2) models. The results showed that olaparib synergized effectively with gemcitabine in in vivo models (Fig. 7e-h and Extended Data Fig. 7e-h). Collectively, these data support the therapeutic potential of olaparib in combination with gemcitabine in PDAC.

\section{Discussion}

PDAC is a life-threatening disease and is projected to be the second most common cause of cancerrelated death by $2030^{14,48}$. The treatment of PDAC remains challenging despite intensive basic and clinical research. In this study, we revealed that elevation METTL16 lead to HRD by blocking DNA end resection. More strikingly, our results show that PDAC cells or PDXs with higher METTL16 expression are more sensitive to PARP inhibitors. Furthermore, greater benefit could be achieved when PARP inhibitors combined with gemcitabine in PDAC. 
DNA end resection is strictly regulated by multiple factors in cells, is the key step that determines the choice between error-free HR and error-prone NHEJ ${ }^{49-51}$. MRE11 has been intensively investigated because of its core role in the MRN complex to process DNA end resection ${ }^{17,19}$. In the current study, we found that a METTL16-RNA complex works as a sponge regulator by attenuating MRE11-mediated end resection. METTL16 is one of the "writers" for $\mathrm{m}^{6} \mathrm{~A}$ modification which specifically methylates RNA containing a consensus sequence of DRACH ${ }^{44,45}$. Unlike METTL3, which plays a role in DNA damage repair by promoting pol $K, \mathrm{BRCA} 1$ or RAD51 recruitment ${ }^{35}$, here we report that METTL16 is involved in a negative regulatory mechanism in DSBs repair. METTL16 inhibits MRE11 nuclease activity in a MTase activity independent manner. Surprisingly, METTL16 alone has no effect on MRE11 nuclease activity. We found that the METTL16-RNA complex plays a key role in MRE11 nuclease activity regulation following DNA damage. An interesting feature of METTL16 protein is its high negative-charge density within the $N$ terminus, which contains the RNA binding domain. Interestingly, the MRE11 DNA binding domain also shows an RNA binding activity. Indeed, we revealed that METTL16 interacts with MRE11 through RNA and leads to inactivation of MRE11 under undamaged conditions. Upon DNA damage, ATM mediated phosphorylation of METTL16 at Ser419 confers negative charge to the $\mathrm{C}$ terminus, leading to a conformational change and the formation of hydrogen bonds with key RNA binding residues in the Nterminal RNA binding domain. This results in decreased RNA binding activity of METTL16. Consequently, released MRE11 can initiate DNA end resection. Therefore, we speculate that the level of METTL16 is strictly regulated and keeps a balance to maintain error-free repair and avoid hyper-end resection in physiology conditions. However, in cancers with elevated expression of METTL16, this balance is disrupted, leading to a decrease of unscheduled end resection and mutagenetic DNA repair.

Taken together, our study demonstrates that PARPi alone or combined with gemcitabine could be potential treatment options for PDAC patients with elevated METTL16 expression. Mechanistically, we uncovered an inhibitory role for RNA and RNA binding proteins in DNA repair, which represents a novel regulatory mode for DNA end resection (Fig. 7i).

\section{Methods}

Tumor Models and PDAC PDX. All manipulations were approved by Institutional Animal Care and Use Committee (IACUC) of Union Hospital, Tongji Medical College, Huazhong University of Science and Technology. Mouse xenografts models were established by transplanting SW1990 METTL16 KO cells or SW1990 METTL16 KO cells re-expressing METTL16 into 6-week-old female BALB/c nude mice. The subcutaneous PDAC PDX models were performed as described ${ }^{52,53}$. Briefly, minced fresh tumor tissue were transplanted into 6-week-old female BALB/c nude mice. After establishment of PDAC-2 and PDAC-3 PDX models, tumor tissue was minced and dissociated into single cells, which were then injected into flanks of nude mice (female, six weeks). Tumors were measured twice a week by caliper and tumor volume was calculated using the formula; Tumor volume $=0.5 \mathrm{~L} \mathrm{~W} \mathrm{~W}$. When tumors measured 100-200 $\mathrm{mm}^{3}$, mice were divided into groups for indicated treatment. 
Cell Lines. HEK293T, U20S, and human PDAC cell lines BxPC-3, PANC-1, SW1990, Capan-2, AsPC-1, HPAFII, HPAC, and CFPAC-1 were purchased from ATCC. The identities of all cell lines were confirmed by the Medical Genome facility at Mayo Clinic Center (Rochester, MN). HEK293T, U2OS, PANC-1, and HPAF-II were maintained in DMEM medium with 10\% FBS. AsPC-1 was maintained in RPMI-1640 medium supplemented with 10\% FBS. SW1990 was maintained in L-15 medium with 10\% FBS. CFPAC-1 was maintained in IMDM medium supplemented with 10\% FBS. Capan-2 was maintained in McCoy's 5a medium supplemented with 10\% FBS. All cells were incubated in a humidified ThermoFisher incubator with $5 \%$ CO2.

Plasmid and antibodies. pCMV-METTL16 vector was purchased from Dharmacon (Clone ID: 6174508) and sub-cloned into pLVX3 and pLVX6 vectors. All METTL16 site mutants and truncated mutants were generated by site-directed mutagenesis. MRE11 wild-type and truncated mutant vectors were kindly provided by Dr Jiadong Wang (Peking University).

Anti-CtIP antibody (ab28448, 1:1000 for western blot) was purchased from Abcam. Anti-METTL16 antibody (A304-192A-T, 1:1000 for western blot), anti-NBS1 antibody (A300-187A, 1:1000 for western blot), and anti-PALB2 antibody (A301-246A, 1:500 for IF) were purchased from Bethyl Laboratories. AntiBrdU antibody (347580, 1:100 for IF) was purchased from BD Biosciences. Anti-pSQ/TQ antibody (2851, 1:1000 for western blot), and anti-Myc antibody $(2276,1: 3000$ for western blot) were purchased from Cell Signaling Technology. Anti-RAD50 antibody (GTX70228, 1:1000 for western blot), and anti-RAD51 (GTX100469, 1:1000 for western blot and IF) antibody were purchased from GeneTex. Anti-RPA32 antibody (MA1-26418, 1:1000 for western blot and IF) was purchased from Invitrogen. Anti-Tubulin antibody (T9026, 1:3000 for western blot), anti-Flag antibody (F3165, 1:3000 for western blot), antiMETTL14 antibody (HPA038002, 1:1000 for western blot), and anti-g-H2AX antibody (05-636, 1:1000 for IF) were purchased from Millipore. Anti-MRE11 antibody (NB100-142, 1:1000 for western blot and1:500 for IF), anti-53BP1 antibody (NB100-304, 1:1000 for western blot) were purchased from Novus. AntiMETTL3 antibody (15073-1-AP, 1:1000 for western blot) was purchased from Proteintech. Anti-GAPDH antibody (sc-32233, 1:3000 for western blot), anti-Actin antibody (sc-47778, 1:3000 for western blot), antiGFP antibody (sc-9996, 1:3000 for western blot), and anti-BRCA1 (6954, 1:1000 for western blot and IF) antibody were purchased from Santa Cruz Biotechnology.

Generation of CRISPR-Cas9 knockout cell lines. The METTL16 knockout was generated in U2OS, SW1990 and PANC-1 cells using the following gRNA: METTL16 gRNA-1: AGAAGATCTGATCGGTCACC; METTL16 gRNA-2: GACAAAAGTACTCTCCGAAG. All METTL16-knockout cells were confirmed by genomic sequence and immunoblotting.

shRNA knockdown. shRNA control (5'-GGGAUACCUAGACGUUCUATT-3'), METTL3 shRNA \#1 (5'CGTCAGTATCTTGGGCAAGTT-3', targeting CDS), METTL3 shRNA \#2 (5'-GCCAAGGAACAATCCATTGTT-3', targeting CDS), METTL14 shRNA \#1 (5'- CCATGTACTTACAAGCCGATA-3', targeting CDS), METTL14 shRNA \#2 (5'- GCTTACAAATAGCAACTACAA-3', targeting CDS), METTL16 shRNA \#1 (5'CCCAAAGTAACGTACACTGAA-3', targeting CDS), METTL16 shRNA \#2 (5'- GTTTGTTATGAGGTGGAGTTT- 
3', targeting 3'UTR), BRCA1 shRNA (5'- GAGTATGCAAACAGCTATAAT-3', targeting CDS), MRE11 shRNA \#1 (5'- TGTTGGTTTGCTGCGTATTAA-3', targeting CDS), and MRE11 shRNA \#2 (5'-

GTTGAGGGAAAGAGCTTATAA-3', targeting 3'UTR) were purchased from Sigma. Lentiviruses were made according to manufacturer's protocol.

Chemicals. The 5-Bromo-2'-deoxyuridine (BrdU, Sigma), ATM inhibitor KU55933 (Sigma), RNase (NEB), DNase (NEB), gemcitabine (Sigma), and olaparib (Selleckchem) were used in this study.

Repair assays. DR-GFP and EJ5-GFP reporter system were established to check repair efficiency. Briefly, $3 \times 10^{5}$ HEK293T cells stably expressing indicated shRNA were seeded in triplicate in each well of six-well plate. Twenty-four hours later, cells in each well were transfected with 1.5I TransIT-X2 mixed with plasmids of 500ng DR-GFP (or 500ng EJ5-GFP), 150ng Mcherry, and 500ng I-Scel. Thirty-six hours after transfection, cells were harvested and analyzed by flow cytometric analysis (FACS) for RFP-positive cells and RFP/GFP both positive cells. Relative percentage of GFP-positive cells was calculated as dividing number of RFP/GFP both positive cells with RFP single-positive cells.

2-D and 3-D soft agar colony formation assay. The colony formation assay and 3-D soft agar assay were performed as previously described ${ }^{54}$. For 2-D colony formation assay, cells (500-1000) were seeded in triplicate in each well of six-well plate. $24 \mathrm{~h}$ later, cells treated with olaparib or gemcitabine as indicated dose, and left for $10-14$ days at $37^{\circ} \mathrm{C}$ to allow colony formation. Colonies were stained with $5 \%$ GIEMSA and counted. For 3-D soft agar colony formation assay, plate bottom layer of agar (1.2\%) first, leaving agar mixture solidifying at room temperature; plate the upper layer of agar containing cells $(5,000-$ $10,000)$, cells treated with olaparib or gemcitabine as indicated dose after $24 \mathrm{~h}$ of seeding. Culture the cells around 3-4 weeks and observe under microscope. Results were normalized to plating efficiencies.

Immunoblotting and Co-immunoprecipitation (Co-IP). Cell lysates, immunoblotting and Coimmunoprecipitation (Co-IP) were performed as previously described. Briefly, cells were lysed with NETN buffer (20 mM Tris- $\mathrm{HCl}, \mathrm{pH}$ 8.0, $100 \mathrm{mM} \mathrm{NaCl}, 1 \mathrm{mM}$ EDTA, 0.5\% Nonidet P-40) containing $50 \mathrm{mM} \beta$ glycerophosphate, $10 \mathrm{mM} \mathrm{NaF}$, and $1 \mathrm{mg} / \mathrm{ml}$ each of pepstatin $A$ and aprotinin. Whole cell lysates were centrifuged at $14000 \mathrm{rpm}$ for 10 minutes. Whole cell lysates were incubated with $2 \mu \mathrm{g}$ of antibody and 20 $\mu$ protein A or protein $\mathrm{G}$ Sepharose beads (Amersham Biosciences) for 2 hours or overnight at $4^{\circ} \mathrm{C}$. The immunocomplexes were then washed with NETN buffer and separated by SDS-PAGE. Immunoblotting was performed following standard procedures.

Soluble and Chromatin Fractions Extraction. Soluble and chromatin fractions extraction was performed as previously described ${ }^{29,55}$. Briefly, U2OS cells treated with IR were harvested at the indicated time. Cells were lased using NETN buffer supplemented with protease and phosphatase cocktail inhibitors (Thermo Fisher) for $15 \mathrm{~min}$ on ice, and centrifuged at $1,200 \mathrm{~g}$ for $10 \mathrm{~min}$. Keep the supernatants as soluble fractions. The pellets were resuspended in Buffer B (Tris- $\mathrm{HCl}(\mathrm{pH} 7.5) 50 \mathrm{mM}, \mathrm{NaCl} 150 \mathrm{mM}, \mathrm{NP}-40$ 1.0\%, SDS $0.1 \%$, Na-deoxycholic acid $0.1 \%$, glycerol $5 \%$, DNase $20 \mathrm{mg} / \mathrm{mg}$, NEM $10 \mathrm{mM}$ ), sonicated about $20 \mathrm{~s}$, 
and centrifuged for 5 min at 12,000g; the supernatant was then transferred to a new tube as chromatin fractions.

Immunofluorescence staining. lonizing radiation induced foci (IRIF) was performed as previously described ${ }^{39,56}$, briefly, cells were cultured on coverslips and treated with 5 Gy or 10 Gy IR followed by recovery for indicated time. Depending on the foci to be stained, cells were then washed in PBS, preextracted with a solution of $20 \mathrm{mM} \mathrm{HEPES} \mathrm{pH} \mathrm{7.4,} 50 \mathrm{mM} \mathrm{NaCl}, 3 \mathrm{mM} \mathrm{MgCl}_{2}, 300 \mathrm{mM}$ Sucrose, and $0.5 \%$ Triton-X for 10 minutes at room temperature, incubated in 3\% paraformaldehyde for 15 minutes, and permeabilized in $0.5 \%$ Triton solution for 5 minutes at room temperature. For RPA32 IRIF, incubation at $-20^{\circ} \mathrm{C}$ in a 1:1 mixture of acetone: methanol was used as fixative. Samples were incubated with primary antibody for overnight at $4^{\circ} \mathrm{C}$. Samples were washed three times and incubated with secondary antibody for $1 \mathrm{~h}$ at room temperature. Cells were stained with DAPI to visualize nuclear DNA. The coverslips were mounted onto glass slides with anti-fade solution and visualized using a Nikon eclipse 80i fluorescence microscope.

Irradiation. Cells were irradiated with $5 \mathrm{~Gy}$ for immunofluorescence studies and $10 \mathrm{~Gy}$ for western blot/ co-immunoprecipitation and BrdU incorporation assays. Typically, cells were processed an hour after irradiation for western blot/co-immunoprecipitation and four hours for immunofluorescence studies unless noted otherwise.

Single-Cell Gel Electrophoresis (SCGE)/Comet Assay. U2OS cells with stable knockdown or overexpression of METTL16 were plated at a density of $2 \times 10^{5}$ cells/well in six-well plates. Cells were analyzed using the Comet SCGE assay kit (Trevigen, 4250-050-K) according to the manufacturer's instructions. Briefly, cells were embedded in low melting agarose on a glass slide and then maintained in lysis buffer for 1 hour at $4^{\circ} \mathrm{C}$. Slides were then incubated with neutral electrophoresis buffer for 60 minutes to allow the DNA to unwind. Subsequently, electrophoresis was carried out for 30 minutes at 300 mA. Next, the slide was immersed in $70 \%$ ethanol for 5 minutes, air dried, and stained with SYBR. For quantification, comets on each gel were observed using a fluorescence microscope. Tail length and tail moment were used to evaluate the degree of DNA damage.

Micronucleus assay. U2OS cells were cultured on coverslips and treated with or without $2 \mathrm{~Gy}$ IR followed by $1 \mathrm{~h}$. Cells were washed in PBS and incubated in 3\% paraformaldehyde for 15 minutes. Cells were stained with DAPI to visualize nuclear DNA. The coverslips were mounted onto glass slides with anti-fade solution and visualized using a Nikon eclipse 80i fluorescence microscope.

RNA pull-down. RNA pull-down was performed as previously described with minor modification ${ }^{57}$. In short, in vitro biotin labeled RNAs were mixed with HEK293T lysate, followed by targeting RNA-protein complexes with streptavidin beads. Western blot analysis was then performed on the co-precipitated proteins.

Recombinant protein production. Purified protein of MRE11/RAD50 (MR) and NBS1 were kindly provided by Dr. Tanya T. Paull (The University of Texas at Austin). Human recombinant protein of METTL16 were 
purchased from Origene (TP308648).

Nuclease reactions. To evaluate the effect of METTL16 on MRN complex exonuclease activity, reactions were set up by premixing the indicated concentrations of MRN complex, METTL16, U6 snRNA and control RNA in 12 I of reaction buffer ( $25 \mathrm{mM}$ MOPS pH 7.0, $20 \mathrm{mM}$ Tris- $\mathrm{HCl}, \mathrm{pH} 8.0,80 \mathrm{mM} \mathrm{NaCl}, 8 \%$ glycerol, 1 $\mathrm{mM}$ DTT, $1 \mathrm{mM}$ ATP, $5 \mathrm{mM} \mathrm{MgCl} 2,1 \mathrm{mM} \mathrm{MnCl} 2$, and $0.2 \mathrm{mg} / \mathrm{mL}$ BSA), followed by the addition of $4 \mathrm{nM}$ dsDNA or overhang DNA substrate. After a 30 minutes incubation at $37^{\circ} \mathrm{C}$, the reaction was terminated by treatment with $0.5 \%$ SDS and $1 \mathrm{mg} / \mathrm{mL}$ proteinase $\mathrm{K}$ for $30 \mathrm{~min}$ at $55^{\circ} \mathrm{C}$. After adding an equal volume of $2 \times$ Novex $^{\text {TM }}$ TBE-Urea Sample Buffer (ThermoFisher Scientific, LC6876), the reaction mixtures were held for $7 \mathrm{~min}$ at $95^{\circ} \mathrm{C}$ before being analyzed in a denaturing polyacrylamide gel containing $7 \mathrm{M}$ urea in TAE buffer. Gels were dried onto filter paper and subjected to phosphorimaging analysis.

Protein-peptide docking. Twenty thousand random conformations of METTL16 peptide PKESGNSQELARG, corresponding to residues 413-425 with phosphorylated Ser419 (pS419), were generated using CYANA $^{58}$, as the fragment library for docking calculations. The structure of METTL16 Nterminal domain (PDB entry 6B92) was modified using ROSETTA (version 3.11) to model a missing loop segment corresponding to residues 188-214. The Rosetta module FlexPepDock ${ }^{59}$ was used for proteinpeptide docking. Before lower resolution pre-optimization docking (20 trajectories each), the METTL16 Nterminal domain and the peptide structures were relaxed, and prepacked. One thousand structures with the highest binding affinity (the lowest I-sc value and highest h-bond number) and the lowest energy were then refined using higher resolution docking protocol with 50 trajectories for each docking. The 100 structures with the lowest energy and highest binding affinity were selected for cluster analysis.

Molecular dynamics simulation. Molecular dynamics (MD) simulations were performed on the best peptide docking model of METTL16 N-terminal domain bound to the pS419 peptide. The same model but with pS419 replaced by Ser419 was used as a control under identical MD conditions. The GROMACS (version 2019.3) software with the all atom CHARMM36 force field ${ }^{60}$ was used for MD simulations and data analysis. The aqueous environment was simulated with explicit TIP3P water molecules in triclinic boxes with a solute-wall minimum distance of $1.5 \mathrm{~nm}$. Charges were neutralized with $\mathrm{Cl}^{-}$atoms, with 150 $\mathrm{mM} \mathrm{NaCl}$ included to mimic physiological conditions. There were 69449 and 69442 atoms in the pS419 and Ser419 peptide systems, respectively. The systems were subjected to steepest decent energy minimization with a maximum force of $200 \mathrm{~kJ} \cdot \mathrm{mol}^{-1} \cdot \mathrm{nm}^{-1}$. The temperature and volume of each system were then equilibrated by running 200 ps of constant volume and constant temperature (NVT) equilibration at $310 \mathrm{~K}$ with a velocity-rescaling thermostat ${ }^{61}$. This was followed by equilibration for $1.0 \mathrm{~ns}$ to a 1.0 bar constant pressure (NPT) bath using the Berendsen weak coupling method. The above equilibration steps were position-restrained on protein and peptide molecules. The MD simulations used periodic boundary conditions with a time step of $2.0 \mathrm{fs}$. The molecular dynamics parameters were set in accordance to the GROMACS user manual for the CHARMM36 force field (http://manual.gromacs.org/documentation/2019/user-guide/force-fields.html). Long-range electrostatic interactions were calculated using the particle mesh Ewald (PME) method ${ }^{62}$ with a Fourier grid spacing of 
$0.12 \mathrm{~nm}$. The LINCS $^{63}$ algorithm was used to control the bond lengths and a leap-frog integrator ${ }^{50}$ was used for all the simulations. Trajectories were written every 20 ps.

Native-polyacrylamide gel (Native-PAGE) analysis. Native PAGE ${ }^{T M}$ Sample Buffer (4X) was used to prepare samples for native (non-denaturing) gel electrophoresis with Native PAGE ${ }^{\mathrm{TM}}$ Novex ${ }^{\circledR}$ Bis-Tris Gels. Lysates were centrifuged at $14,000 \times \mathrm{g}$ for 60 minutes at $4^{\circ} \mathrm{C}$. Native PAGE ${ }^{\mathrm{TM}} 5 \% \mathrm{G}-250$ Sample Additive was added to samples and then electrophoresis was immediately performed with a nativepolyacrylamide gel. The following steps were the same as standard immunoblotting.

Duo-link in situ assay. Duo-link in situ assay was performed as previously described ${ }^{64}$. Briefly, U2OS cells were transfected with WT, S419A, S419D, and 2RE GFP-METTL16-Flag. Cells were then washed in PBS, pre-extracted with a solution of $20 \mathrm{mM}$ HEPES pH 7.4, $50 \mathrm{mM} \mathrm{NaCl}, 3 \mathrm{mM} \mathrm{MgCl}_{2}, 300 \mathrm{mM}$ Sucrose, and $0.5 \%$ Triton- $X$ for 10 minutes at room temperature, incubated in $3 \%$ paraformaldehyde for 15 minutes, and permeabilized in $0.5 \%$ Triton solution for 5 minutes at room temperature. Samples were incubated with primary antibody overnight at $4^{\circ} \mathrm{C}$. The slides were incubated with a mixture of primary antibodies containing anti-GFP rabbit monoclonal antibody (targeting N-terminus region of METTL16) and anti-Flag mouse polyclonal sera (targeting C-terminus region of METTL16). Control experiments used no primary antibody. Intramolecular interactions were detected and visualized by proximity ligation assay and rolling circle amplification using duo-link in situ kit according to the manufacturer's instructions (Sigma).

Tissue microarray, immunohistochemistry and H\&E staining. The tissue microarrays of PDAC specimen were purchased from Superchip Incorporation. The immunohistochemistry (IHC) and H\&E staining were performed according to a previously published study ${ }^{65}$. Antibodies were diluted at appropriate concentrations. The IHC staining was scored based on staining intensity and ratio of positive cells as described previously. Tumor cell staining score was determined separately by two independent experts under the same conditions. IHC and H\&E slides were observed with Olympus BX51 microscope and Olympus DP73 CCD photographic system.

Survival Analysis. The effects of METTL3, METTL14, and METTL16 expression on the survival of patients with PDAC were performed by SPSS. Public TCGA survival data in PDAC patients were analyzed using The Human Protein Atlas online tool.

Statistical analysis. Data in bar or line graphs are presented as means \pm s.d. of at least three independent experiments. All western blot assays shown here were successfully repeated at least three times. Comparisons were carried out with ANOVA or unpaired Student's t-test $\left({ }^{\star} p<0.05,{ }^{\star \star} p<0.01,{ }^{\star \star \star} p<0.001\right.$, $\star \star \star \star p<0.0001)$.

\section{Declarations}

\section{Acknowledgements}


This research was supported by funding from the National Natural Science Foundation of China $(81874184,82074191)$ and the Mayo Foundation. J.A.K. is supported by NIH T32 GM65841. We thank Dr. Jiadong Wang for MRE11 WT and mutant vectors.

\section{Author contributions}

Z.L., K.T., and J.Y. conceived and designed the study. X.Z. and F.Z. performed most of the experiments and wrote the manuscript. G.C. and G.M. performed the molecular dynamics simulations. M.D. and J.H. helped with the nuclease reaction assay. W.K. helped to analyze the ionizing radiation induced foci data. J.K., K.L., and K.T. reviewed and edited the manuscript. R.D. and T.P. provided support for generating protein purification. H.G., C.Z. and Q.Z. helped with flow cytometric analysis. Y.S. and P.Y. carried out xenograft experiment. Y.Z. and X.T. provided technical expertise with RNA pull-down assay. J.H. and G.G. helped with vectors construction.

\section{Competing interests}

The authors declare no competing interests.

\section{References}

1. Brand $\mathrm{R}$, et al. Prospective study of germline genetic testing in incident cases of pancreatic adenocarcinoma. Cancer 124, 3520-3527 (2018).

2. Li JT, et al. BCAT2-mediated BCAA catabolism is critical for development of pancreatic ductal adenocarcinoma. Nat Cell Biol 22, 167-174 (2020).

3. Biffi G, et al. IL1-Induced JAK/STAT Signaling Is Antagonized by TGF $\beta$ to Shape CAF Heterogeneity in Pancreatic Ductal Adenocarcinoma. Cancer Discov 9, 282-301 (2019).

4. Principe DR, et al. Long-Term Gemcitabine Treatment Reshapes the Pancreatic Tumor Microenvironment and Sensitizes Murine Carcinoma to Combination Immunotherapy. Cancer Res 80, 3101-3115 (2020).

5. Wang Y, et al. A Preclinical Trial and Molecularly Annotated Patient Cohort Identify Predictive Biomarkers in Homologous Recombination-deficient Pancreatic Cancer. Clin Cancer Res 26, 5462-5476 (2020).

6. Costa-Silva B, et al. Pancreatic cancer exosomes initiate pre-metastatic niche formation in the liver. Nat Cell Biol 17, 816-826 (2015).

7. Sehdev A, et al. Germline and Somatic DNA Damage Repair Gene Mutations and Overall Survival in Metastatic Pancreatic Adenocarcinoma Patients Treated with FOLFIRINOX. Clin Cancer Res 24, 62046211 (2018). 
8. Asher G, et al. Poly(ADP-ribose) polymerase 1 participates in the phase entrainment of circadian clocks to feeding. Cell 142, 943-953 (2010).

9. Seufferlein T \& Mayerle J. Pancreatic cancer in 2015: Precision medicine in pancreatic cancer-fact or fiction? Nat Rev Gastroenterol Hepatol 13, 74-75 (2016).

10. Bryant HE, et al. Specific killing of BRCA2-deficient tumours with inhibitors of poly(ADP-ribose) polymerase. Nature 434, 913-917 (2005).

11. Farmer $\mathrm{H}$, et al. Targeting the DNA repair defect in BRCA mutant cells as a therapeutic strategy. Nature 434, 917-921 (2005).

12. Kaufman B, et al. Olaparib monotherapy in patients with advanced cancer and a germline BRCA $1 / 2$ mutation. J Clin Oncol 33, 244-250 (2015).

13. Rouleau M, et al. PARP inhibition: PARP1 and beyond. Nat Rev Cancer 10, 293-301 (2010).

14. Golan T, et al. Maintenance Olaparib for Germline BRCA-Mutated Metastatic Pancreatic Cancer. $N$ Engl J Med 381, 317-327 (2019).

15. Yurgelun MB, et al. Germline cancer susceptibility gene variants, somatic second hits, and survival outcomes in patients with resected pancreatic cancer. Genet Med 21, 213-223 (2019).

16. Ryan DP, Hong TS \& Bardeesy N. Pancreatic adenocarcinoma. N Engl J Med 371, 1039-1049 (2014).

17. Jachimowicz RD, et al. UBQLN4 Represses Homologous Recombination and Is Overexpressed in Aggressive Tumors. Cell 176, 505-519 (2019).

18. Barber LJ, et al. RTEL1 maintains genomic stability by suppressing homologous recombination. Cell 135, 261-271 (2008).

19. Bai Y, et al. C1QBP Promotes Homologous Recombination by Stabilizing MRE11 and Controlling the Assembly and Activation of MRE11/RAD50/NBS1 Complex. Mol Cell 75, 1299-1314 (2019).

20. Wang Q, et al. Rad17 recruits the MRE11-RAD50-NBS1 complex to regulate the cellular response to DNA double-strand breaks. EMBO J 33, 862-877 (2014).

21. Kanakkanthara A, et al. ZC3H18 specifically binds and activates the BRCA1 promoter to facilitate homologous recombination in ovarian cancer. Nat Commun 10, 4632-4636 (2019).

22. He YJ, et al. DYNLL1 binds to MRE11 to limit DNA end resection in BRCA1-deficient cells. Nature 563, 522-526 (2018).

23. Stracker TH \& Petrini JH. The MRE11 complex: starting from the ends. Nat Rev Mol Cell Biol 12, 90103 (2011). 
24. Hopfner KP, et al. Structural biochemistry and interaction architecture of the DNA double-strand break repair Mre11 nuclease and Rad50-ATPase. Cell 105, 473-485 (2001).

25. Lobachev KS, Gordenin DA \& Resnick MA. The Mre11 complex is required for repair of hairpin-capped double-strand breaks and prevention of chromosome rearrangements. Cell 108, 183-193 (2002).

26. Buis $\mathrm{J}$, et al. Mre11 nuclease activity has essential roles in DNA repair and genomic stability distinct from ATM activation. Cell 135, 85-96 (2008).

27. Li Z, et al. Plk1 phosphorylation of Mre11 antagonizes the DNA damage response. Cancer Research 77, 3169-3180 (2017).

28. Jachimowicz RD, et al. UBQLN4 represses homologous recombination and is overexpressed in aggressive tumors. Cell 176, 505-519 (2019).

29. Bai Y, et al. C1QBP Promotes Homologous Recombination by Stabilizing MRE11 and Controlling the Assembly and Activation of MRE11/RAD50/NBS1 Complex. Mol Cell 75, 1299-1314 (2019).

30. He YJ, et al. DYNLL1 binds to MRE11 to limit DNA end resection in BRCA1-deficient cells. Nature 563, 522-526 (2018).

31. Liu J, et al. m6A mRNA methylation regulates AKT activity to promote the proliferation and tumorigenicity of endometrial cancer. Nat cell biol 20, 1074-1083 (2018).

32. Barranco C. Viral infection linked to $m 6$ A alterations in host mRNAs. Nat Rev Mol Cell Biol 21, 64-65 (2020).

33. Lin S \& Gregory RI. Methyltransferases modulate RNA stability in embryonic stem cells. Nat cell biol 16, 129-131 (2014).

34. Xiang $Y$, et al. RNA $m 6$ A methylation regulates the ultraviolet-induced DNA damage response. Nature 543, 573-576 (2017).

35. Zhang C, et al. METTL3 and N6-Methyladenosine Promote Homologous Recombination-Mediated Repair of DSBs by Modulating DNA-RNA Hybrid Accumulation. Mol Cell 79, 425-442 (2020).

36. Kleger A, Perkhofer L \& Seufferlein T. Smarter drugs emerging in pancreatic cancer therapy. Ann Oncol 25, 1260-1270 (2014).

37. Helms E, Onate MK \& Sherman MH. Fibroblast Heterogeneity in the Pancreatic Tumor Microenvironment. Cancer Discov 10, 648-656 (2020).

38. Perkhofer L, et al. ATM Deficiency Generating Genomic Instability Sensitizes Pancreatic Ductal Adenocarcinoma Cells to Therapy-Induced DNA Damage. Cancer Res 77, 5576-5590 (2017). 
39. Nowsheen $\mathrm{S}$, et al. L3MBTL2 orchestrates ubiquitin signalling by dictating the sequential recruitment of RNF8 and RNF168 after DNA damage. Nat Cell Biol 20, 455-464 (2018).

40. Mukherjee B, Tomimatsu N \& Burma S. Immunofluorescence-based methods to monitor DNA end resection. Methods Mol Biol 1292, 67-75 (2015).

41. D'Amours D \& Jackson SP. The Mre11 complex: at the crossroads of dna repair and checkpoint signalling. Nat Rev Mol Cell Bio/ 3, 317-327 (2002).

42. Williams RS, et al. Mre11 dimers coordinate DNA end bridging and nuclease processing in doublestrand-break repair. Cell 135, 97-109 (2008).

43. Mendel M, et al. Methylation of structured RNA by the m6A writer METTL16 is essential for mouse embryonic development. Mol cell 71, 986-1000 (2018).

44. Pendleton KE, et al. The U6 snRNA m6A methyltransferase METTL16 regulates SAM synthetase intron retention. Cell 169, 824-835 (2017).

45. Doxtader KA, et al. Structural basis for regulation of METTL16, an S-adenosylmethionine homeostasis factor. Mol cell 71, 1001-1011 (2018).

46. Golan T, et al. Recapitulating the clinical scenario of BRCA-associated pancreatic cancer in preclinical models. Int J Cancer 143, 179-183 (2018).

47. Perkhofer $L$, et al. DNA damage repair as a target in pancreatic cancer: state-of-the-art and future perspectives. Gut 70, 606-617 (2021).

48. Humpton TJ, et al. Oncogenic KRAS Induces NIX-Mediated Mitophagy to Promote Pancreatic Cancer. Cancer Discov 9, 1268-1287 (2019).

49. Huertas $\mathrm{P}$, et al. CDK targets Sae2 to control DNA-end resection and homologous recombination. Nature 455, 689-692 (2008).

50. Broderick R, et al. EXD2 promotes homologous recombination by facilitating DNA end resection. Nat Cell Biol 18, 271-280 (2016).

51. Zhao $F$, et al. DNA end resection and its role in DNA replication and DSB repair choice in mammalian cells. Exp Mol Med 52, 1705-1714 (2020).

52. Rodrigues G, et al. Tumour exosomal CEMIP protein promotes cancer cell colonization in brain metastasis. Nat Cell Bio/ 21, 1403-1412 (2019).

53. Yoshida GJ. Applications of patient-derived tumor xenograft models and tumor organoids. $J$ Hematol Oncol 13, 4 (2020). 
54. Zhang Y, et al. BAP1 links metabolic regulation of ferroptosis to tumour suppression. Nat Cell Bio/ 20, 1181-1192 (2018).

55. Parvin S, et al. LMO2 Confers Synthetic Lethality to PARP Inhibition in DLBCL. Cancer Cell 36, 237249 (2019).

56. Yuan J, et al. USP10 regulates p53 localization and stability by deubiquitinating p53. Cel/ 140, 384396 (2010).

57. Barnes $C \&$ Kanhere A. Identification of RNA-Protein Interactions Through In Vitro RNA Pull-Down Assays. Methods Mol Biol 1480, 99-113 (2016).

58. Güntert P. Automated NMR structure calculation with CYANA. Methods Mol Bio/ 278, 353-378 (2004).

59. Raveh B, et al. Rosetta FlexPepDock ab-initio: simultaneous folding, docking and refinement of peptides onto their receptors. PLoS One 6, e18934 (2011).

60. Huang J \& Mackerell AD. CHARMM36 all-atom additive protein force field: validation based on comparison to NMR data. J Comput Chem 34, 2135-2145 (2013).

61. Bussi G, Donadio D \& Parrinello M. Canonical sampling through velocity rescaling. J Chem Phys 126 , 014101 (2007).

62. Linse B \& Linse P. Tuning the smooth particle mesh Ewald sum: application on ionic solutions and dipolar fluids. J Chem Phys 141, 184114 (2014).

63. Hess B. P-LINCS: A Parallel Linear Constraint Solver for Molecular Simulation. J Chem Theory Comput 4, 116-122 (2008).

64. Liu T, et al. A divergent role of the SIRT1-TopBP1 axis in regulating metabolic checkpoint and DNA damage checkpoint. Mol Cell 56, 681-695 (2014).

65. Yan Y, et al. The deubiquitinase USP36 Regulates DNA replication stress and confers therapeutic resistance through PrimPol stabilization. Nucleic Acids Res 48, 12711-12726 (2020).

\section{Figures}


a

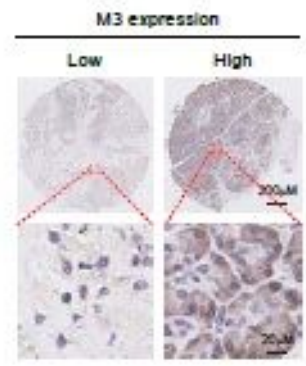

b
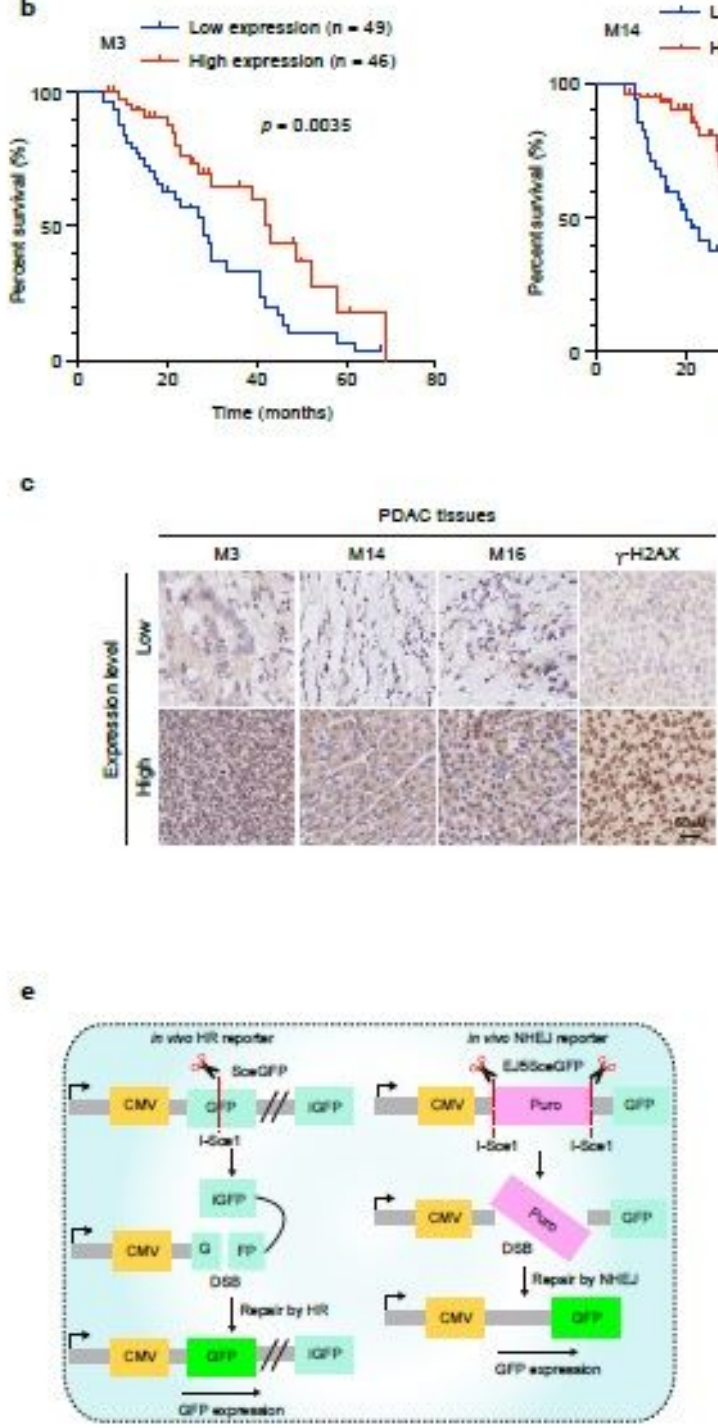
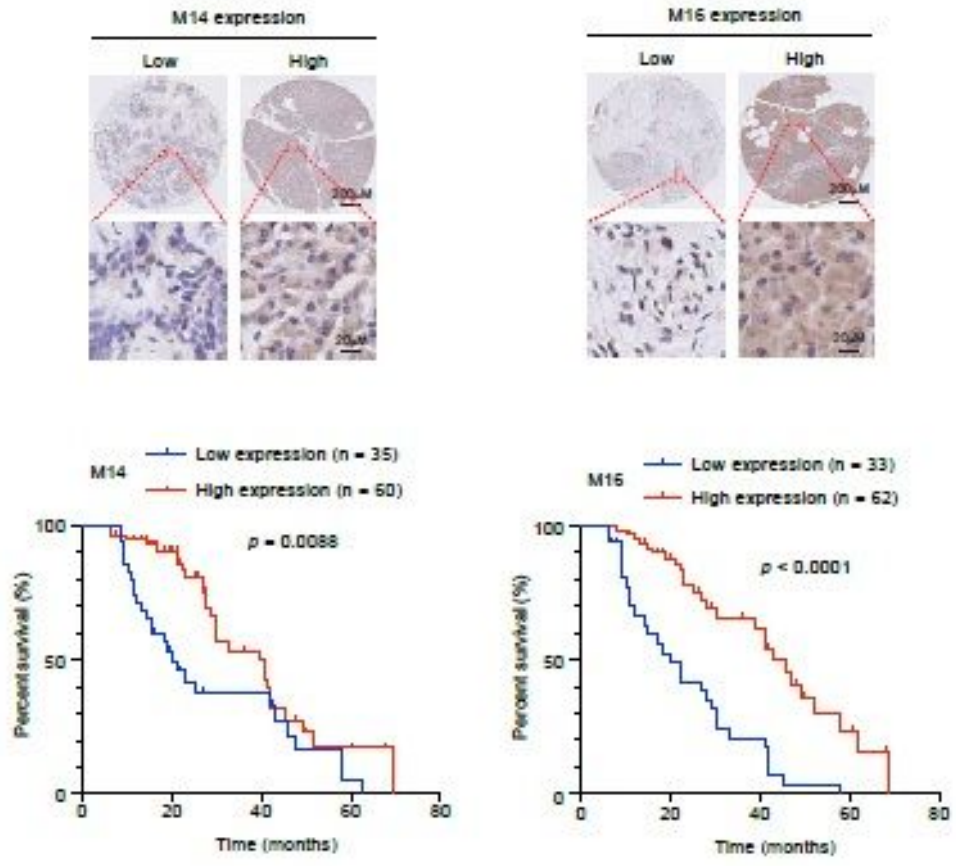

d

\begin{tabular}{|c|c|c|c|c|c|}
\hline $\begin{array}{l}\text { Coms } \\
\text { PDA }\end{array}$ & $\begin{array}{l}\text { syon or M3 } \\
\text { tisgues in }\end{array}$ & 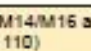 & ${ }_{10} r+12 A X$ & xpression & \\
\hline & & & $2 \mu x$ & & \\
\hline & & $\operatorname{Los}(42)$ & High (68) & pralue & Pearson $\mathrm{F}$ \\
\hline W & Low (59) & 12 & 47 & L & ח? \\
\hline & High (51) & 30 & 21 & & -0.395 \\
\hline & Low (38) & 14 & 24 & 0.9335 & דרירחה \\
\hline & Hilgh (72) & 28 & 44 & & \\
\hline$M$ & Low (35) & 29 & 6 & $\leq=0 \mathrm{con}$ & 0678 \\
\hline & Hlgh (75) & 13 & 62 & & \\
\hline
\end{tabular}

f

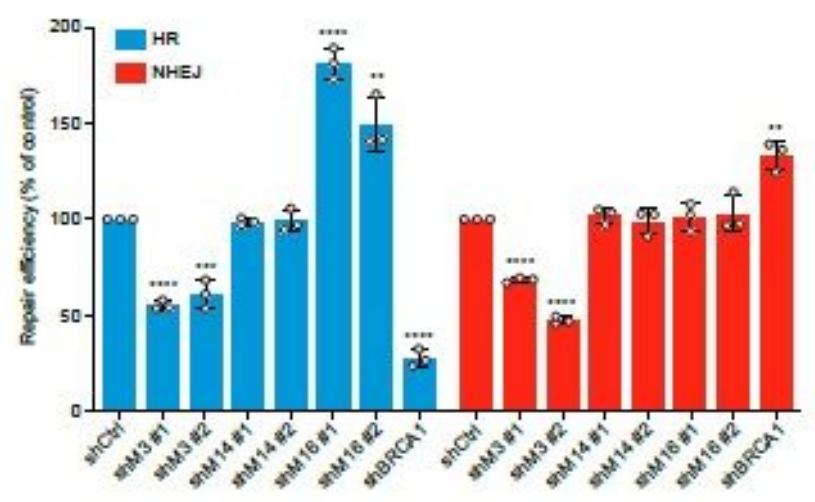

\section{Figure 1}

METTL16 expression indicates favorable overall survival with accumulation of DSBs in PDAC. a, Representative immunohistochemistry (IHC) micrographs showing METTL3, METTL14, or METTL16 low and high expression on tissue microarray of pancreatic ductal adenocarcinoma (PDAC) specimens. Scale bars are indicated. b, Kaplan-Meier survival curves for overall survival of PDAC patients with low and high expression of METTL3, METTL14, or METTL16 in tissue microarray (log rank test; p value are indicated). 
c, Representative IHC micrographs showing METTL3, METTL14, METTL16, and g-H2AX expression (low and high), respectively. Scale bar is indicated. d, METTL3, METTL14, and METTL16 expression correlate with g-H2AX levels in tissue microarray of PDAC samples. Protein levels of METTL3, METTL14, METTL16, and g-H2AX were quantified in PDAC specimens. ( $\chi 2$ test; $p$ values are indicated). e, Schematic of the DR-GFP/EJ5-GFP reporter system. $f$, DNA repair efficiency after knockdown of indicated proteins with shRNAs in HEK293T cells. shBRCA1 was used as a positive control. Data are presented as mean \pm SD from three independent experiments (student's t test; ${ }^{* \star} p<0.01$, ${ }^{\star \star \star} p<0.001,{ }^{\star \star \star *} p<0.0001$ ).

a

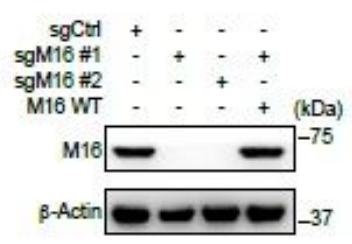

b

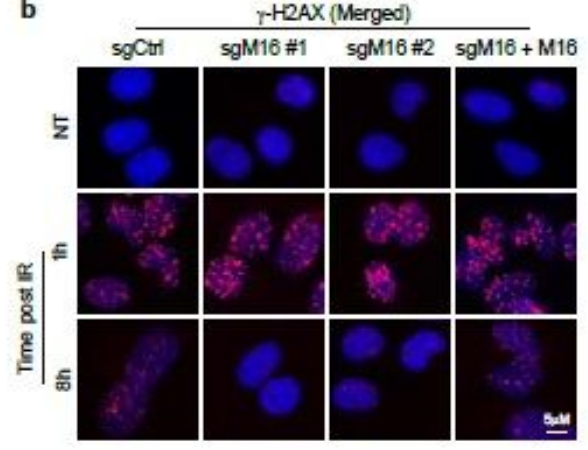

d
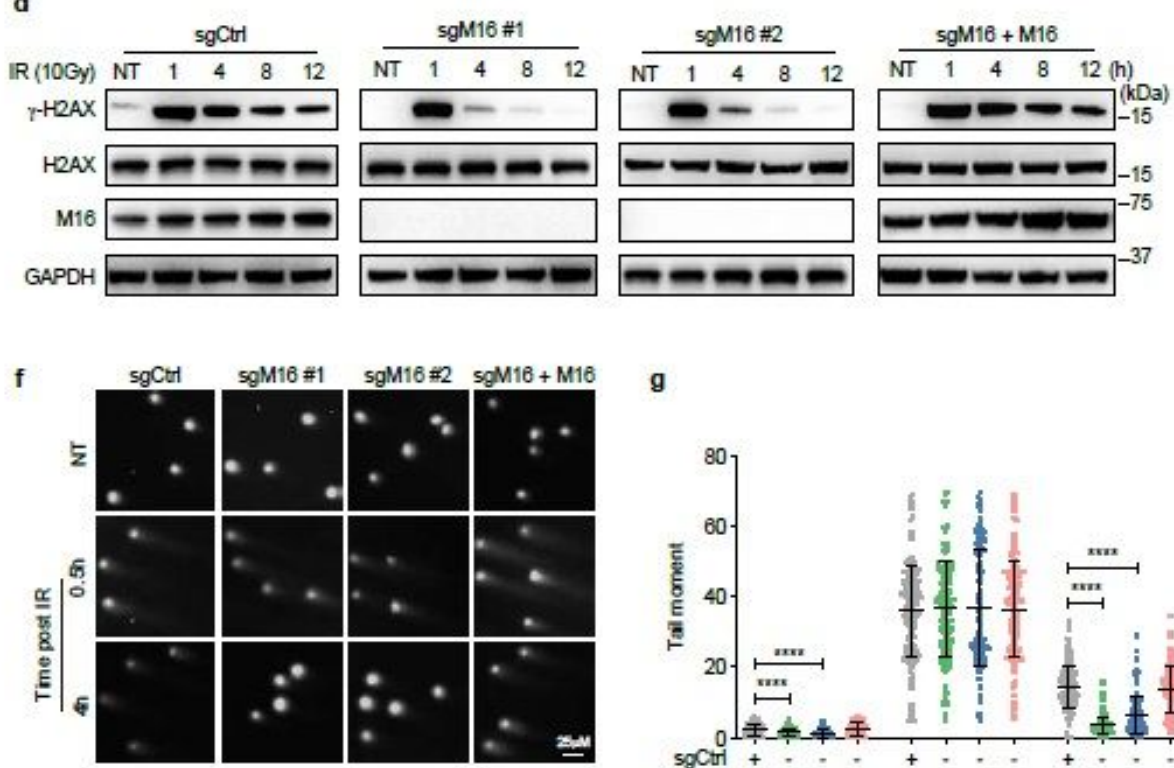

g

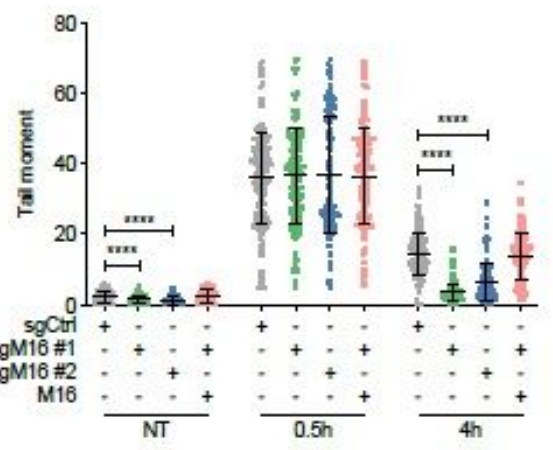

i

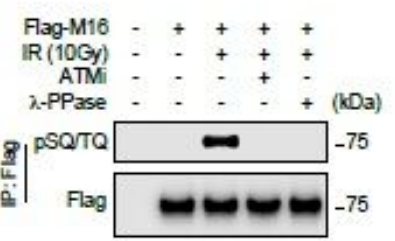

j

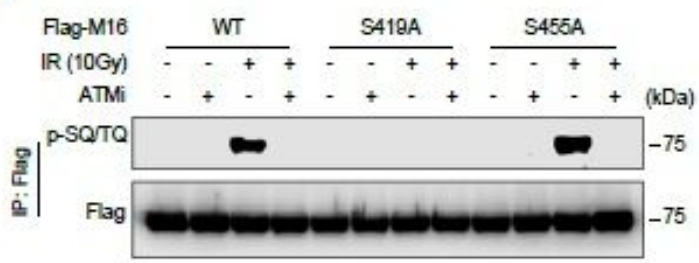

c

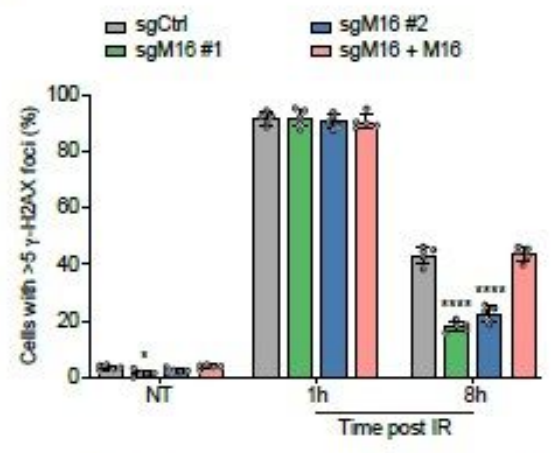

e

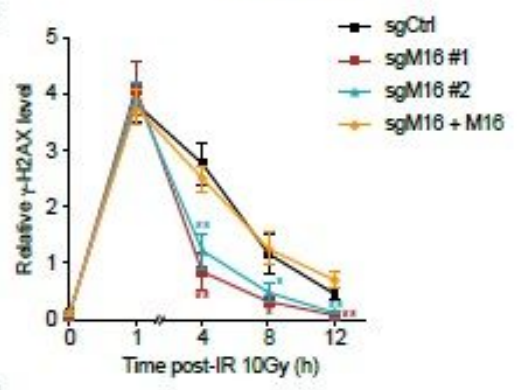

h

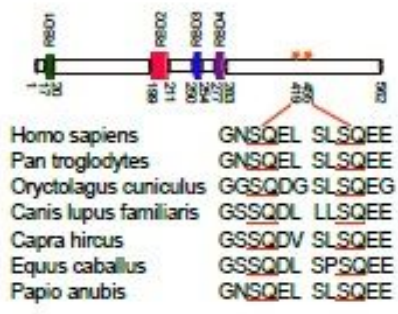

k

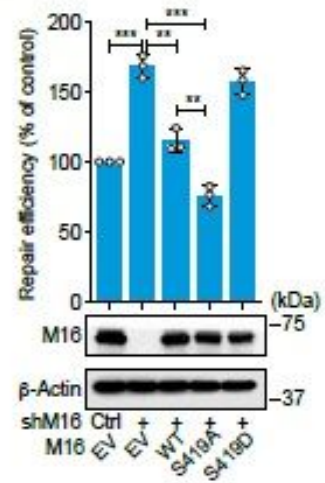

Figure 2 
METTL16 inhibits HR mediating by ATM phosphorylation. a, Immunoblot of METTL16 in U2OS cells after KO by two independent sgRNAs and re-expression of WT METTL16. b-Actin was used as a loading control. b and c, Representative images b and quantification c of g-H2AX foci formation in the indicated U2OS cells without irradiation (IR) or at $1 \mathrm{~h}$ and $8 \mathrm{~h}$ after IR with $5 \mathrm{~Gy}$. At least 150 cells were analyzed for each datum point. Data are presented as mean \pm SD from five independent experiments (student's $t$ test; $\left.{ }^{*} p<0.05,{ }^{* \star *} \mathrm{p}<0.0001\right)$. Scale bar is indicated. $d$, Comparison of IR-induced $g-H 2 A X$ levels in the indicated U2OS cells. Immunoblot detection of $\mathrm{g}-\mathrm{H} 2 \mathrm{AX}$ was collected at the indicated time point after IR with $10 \mathrm{~Gy}$. NT, no treatment. e, Quantitative analysis of the relative levels of g-H2AX normalized to H2AX loading control by ImageJ software. Data are presented as mean \pm SD from three independent experiments (two-way ANOVA; ${ }^{*} p<0.05$, ${ }^{\star *} p<0.01$ ). $f$ and $g$, Representative images $f$ and quantification $\mathrm{g}$ of neutral comet assay in the indicated U2OS cells without IR or at $0.5 \mathrm{~h}$ and $4 \mathrm{~h}$ after IR with $5 \mathrm{~Gy}$. The tail moment was analyzed using the CometScore software. At least 200 cells were analyzed for each datum point. Data are presented as mean \pm SD from five independent experiments (student's $t$ test; ${ }^{* \star \star \star p}$ $<0.0001)$. Scale bar is indicated. $h, A$ schematic structure and amino acid sequence of METTL16 in different species containing two conserved SQ residues which might be phosphorylated by ATM/ATR. i, HEK293T cells were transfected with empty vector or Flag-METTL16. Cells were pretreated with DMSO or ATM inhibitor (KU55933, $10 \mathrm{mM}$ ) for $1 \mathrm{~h}$ and then were further treated with or without IR (10Gy) for $1 \mathrm{~h}$. Cell lysates were treated with or without I-PPase (P0753S, 1000 units) for $1 \mathrm{~h}$ and then were immunoprecipitated with Flag beads, and immunoblotted with the indicated antibodies. j, Identification of ATM-phosphorylation sites in METTL16 by immunoprecipitation with Flag after transfected METTL16 WT, S419A, and S455A vectors in HEK293T cells with indicated treatment. $k$, HR repair efficiency in the indicated HEK293T cells. Western blot below showing expression level of METTL16. Data are presented as mean \pm SD from three independent experiments (student's $t$ test; ${ }^{\star \star} p<0.01, \star \star \star p<0.001$ ). 
a
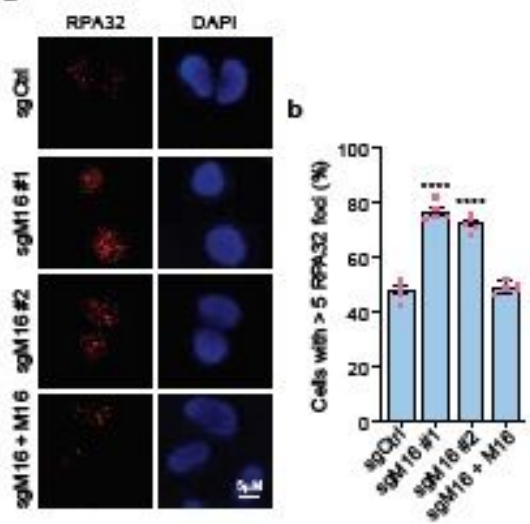

c

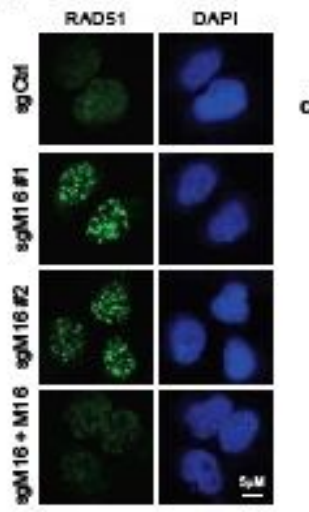

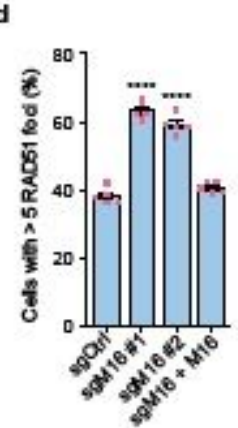

h
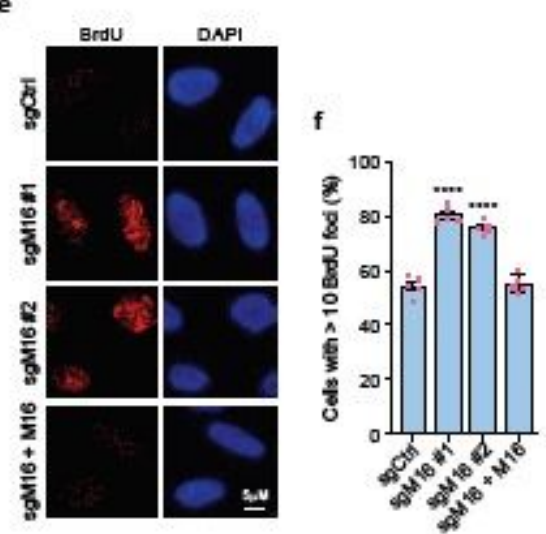
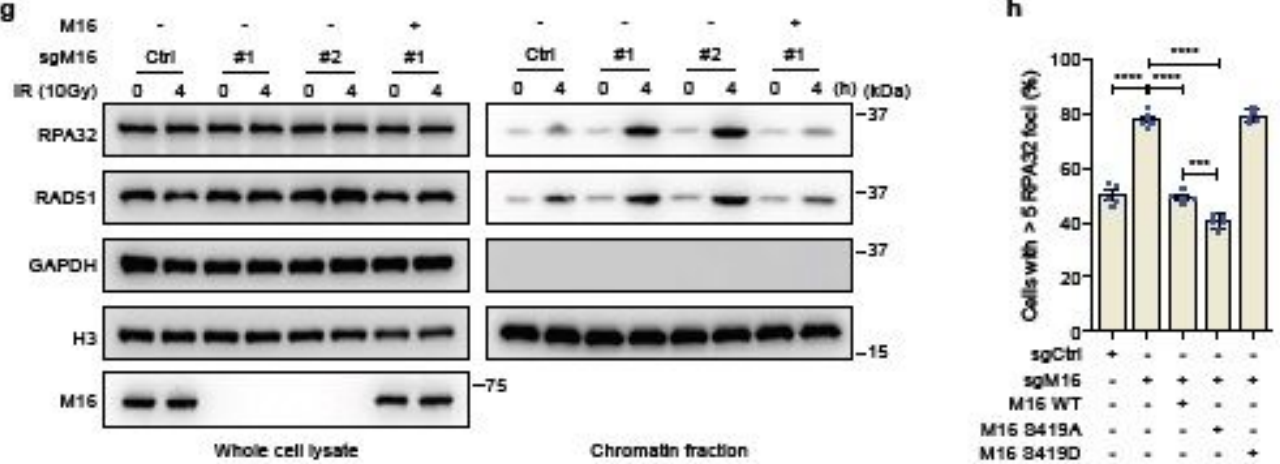

i

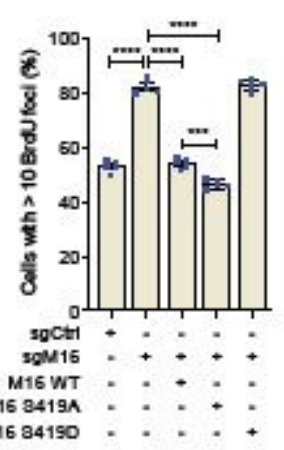

j

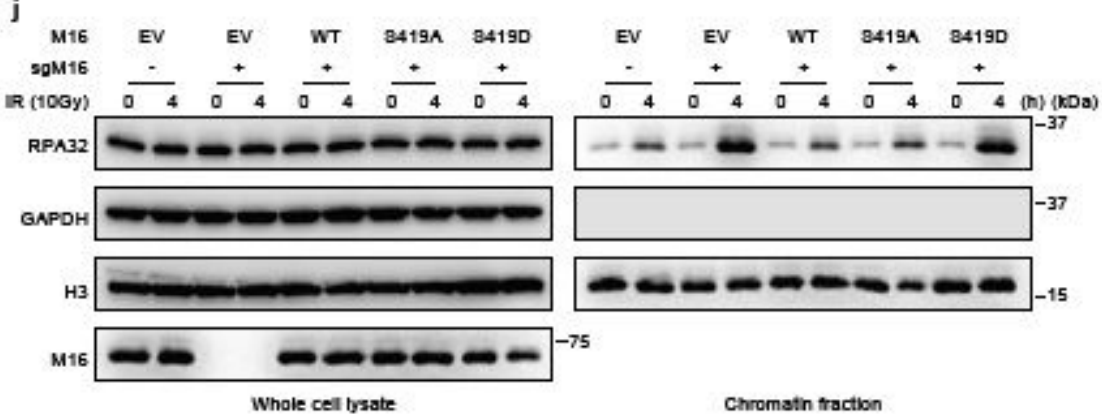

k

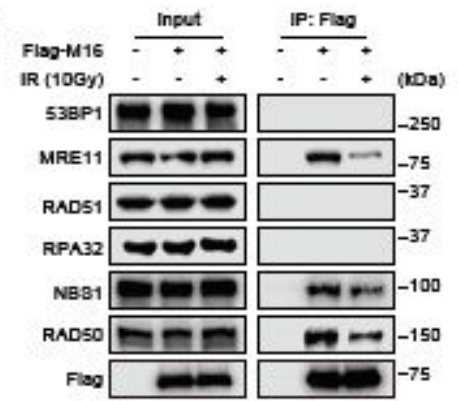

I

m
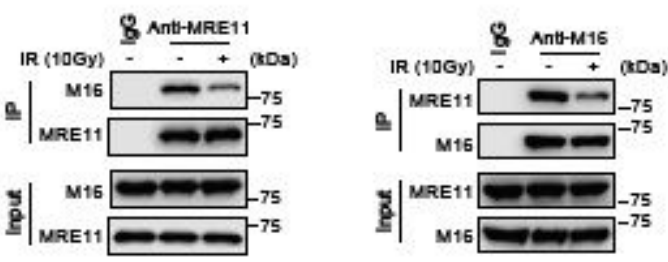

n
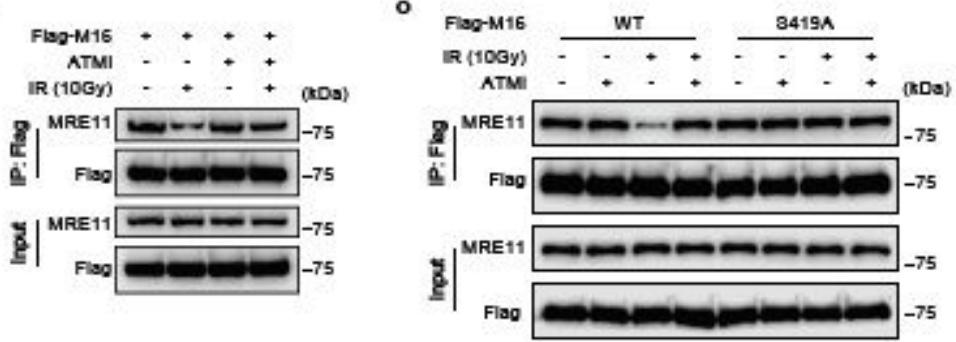

\section{Figure 3}

METTL16 represses DNA end resection in a MRE11 dependent manner. a-f, Representative images a, c, and $e$ and quantification $b, d$, and $f$ of RPA32, RAD51, and BrdU foci in the indicated U20S cells. RPA32 and RAD51 foci are shown at $4 \mathrm{~h}$ after IR with $5 \mathrm{~Gy}$. BrdU foci are shown at $1 \mathrm{~h}$ after IR with $10 \mathrm{~Gy}$. At least 150 cells were analyzed. Data are presented as mean \pm SD from five independent experiments (student's t test; ${ }^{* \star * *} p<0.0001$ ). Scale bars are indicated. $g$, Western blots with total or chromatin- 
enriched extracts from indicated U2OS cells without IR or at $4 \mathrm{~h}$ after IR with $10 \mathrm{~Gy}$. $\mathrm{h}$ and i, Quantification of RPA32 $\mathrm{h}$ and BrdU $\mathrm{i}$ foci in the indicated U20S cells. At least 150 cells were analyzed. Data are presented as mean \pm SD from five independent experiments (student's $t$ test; ${ }^{* \star *} p<<0.0001$ ). j, Western blots with total or chromatin-enriched extracts from indicated U2OS cells without IR or at $4 \mathrm{~h}$ after IR with 10 Gy. k, HEK293T cells were transfected with Flag-METTL16 following IR with $10 \mathrm{~Gy}$. Cell lysates were subjected to immunoprecipitation with Flag beads and immunoblotted with the indicated antibodies. Input of cell lysates were shown at left panel. I and m, HEK293T cell lysates were subjected to immunoprecipitation with control immunoglobulin G (IgG), anti-MRE11, and anti-METTL16 antibodies and immunoblotted with the indicated antibodies. n, HEK293T cells were transfected with Flag-METTL16. Cells were pretreated with DMSO or ATM inhibitor (KU55933, $10 \mathrm{mM})$ for $1 \mathrm{~h}$ and then were further treated with or without IR (10 Gy) with a $1 \mathrm{~h}$ recovery. Cell lysates were subjected to immunoprecipitation with Flag beads and immunoblotted with the indicated antibodies. O, HEK293T cells were transfected with Flag-METTL16 WT or S419A vectors following indicated treatment. Cell lysates were subjected to immunoprecipitation with Flag beads and immunoblotted with the indicated antibodies. 

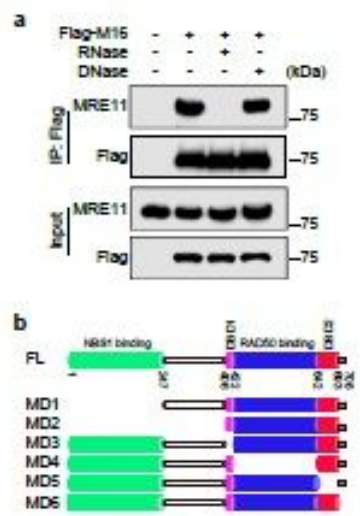

f

Bloon-Fin COI U6 InFNA

Fog

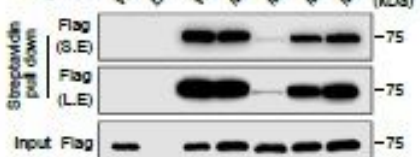

Inout Fos $-\quad-\infty-\infty-75$

h
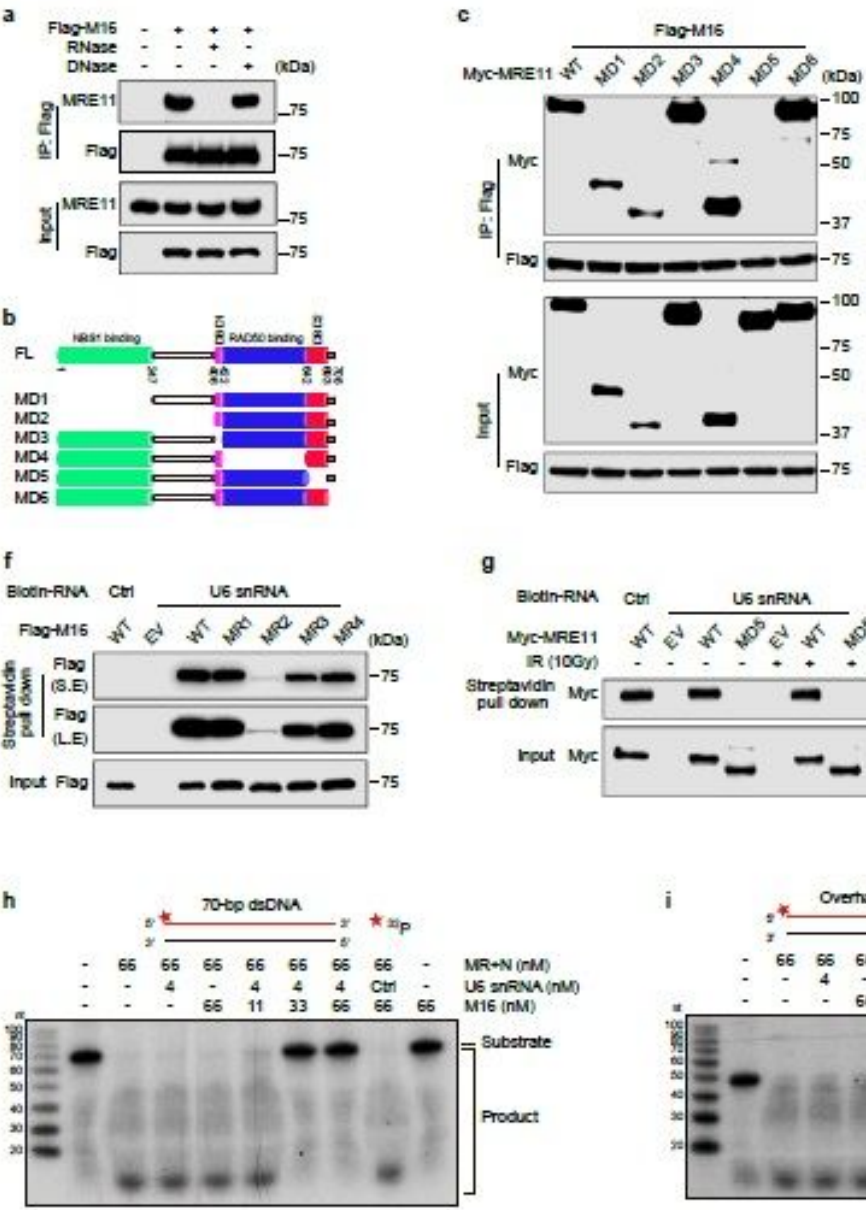

g

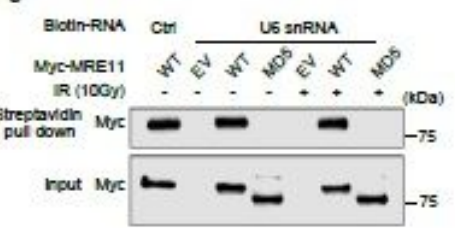

i
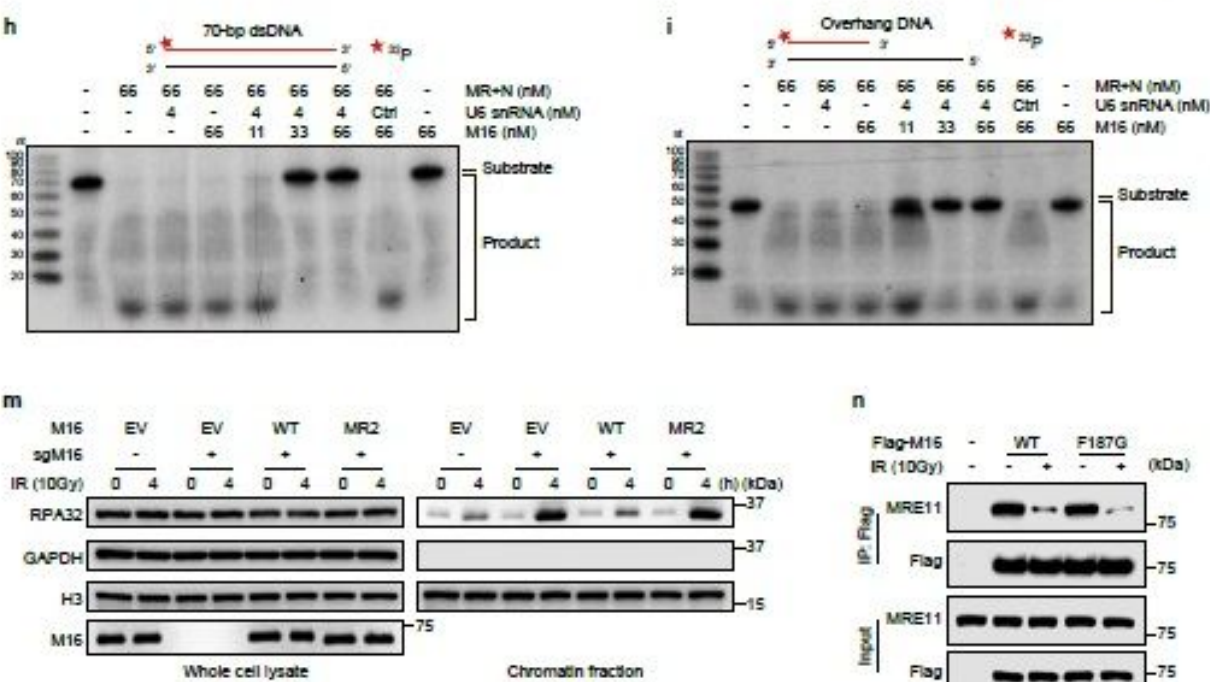

n

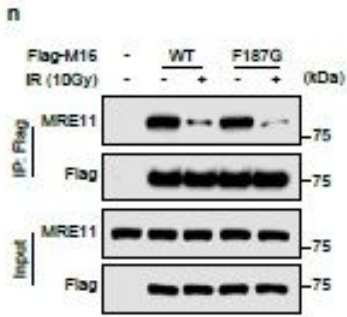

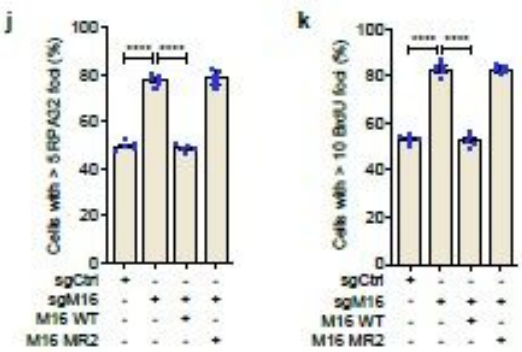

d
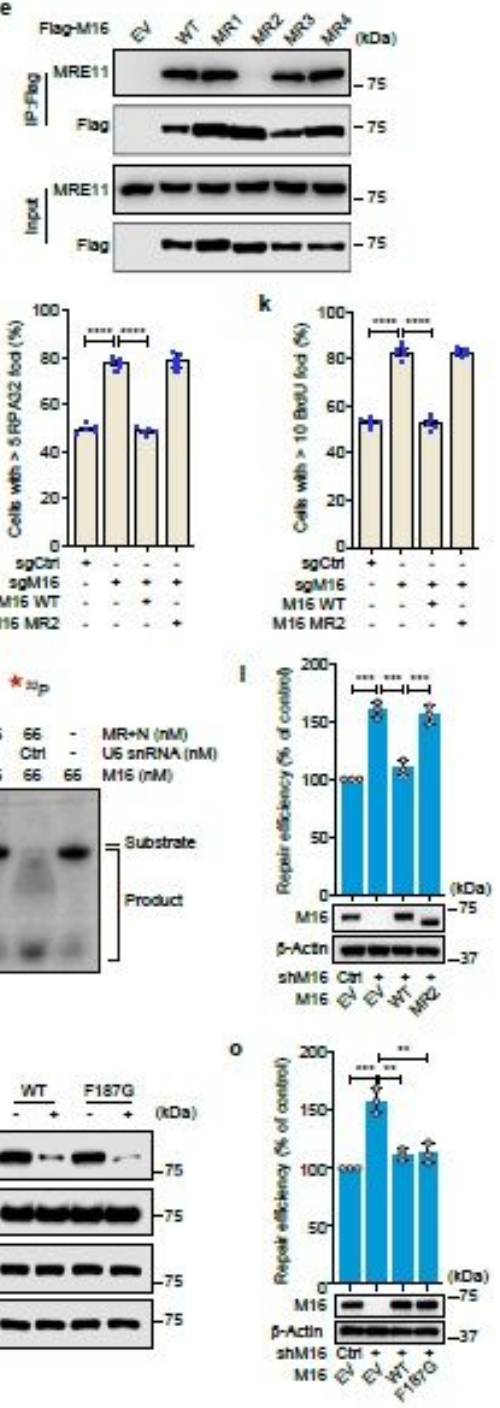

s
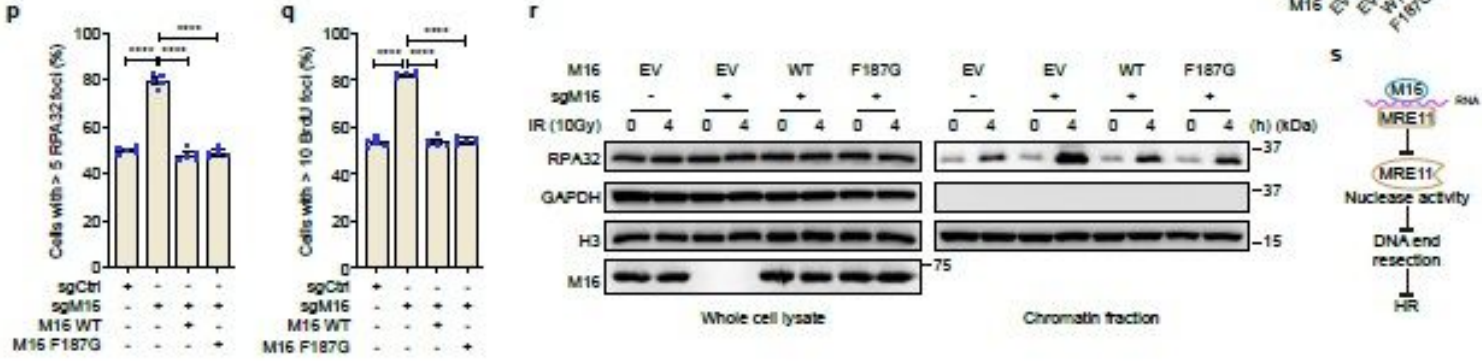

\section{Figure 4}

RNA orchestrates METTL16-mediated MRE11 inactivation. a, HEK293T cells were transfected with empty vector or Flag-METTL16. Cell lysates were pretreated with DNase (M0303S, 10 units) or RNase (T3018L, $100 \mathrm{mg}$ ) and then subjected to immunoprecipitation with Flag beads and immunoblotted with the indicated antibodies. b, A schematic structure of MRE11 truncation constructs. c, HEK293T cells were transfected with Flag-METTL16 and indicated MRE11 vectors. Cell lysates were subjected to 
immunoprecipitation with Flag beads and immunoblotted with the indicated antibodies. d, A schematic structure of METTL16 truncation constructs. e, HEK293T cells were transfected with empty vector or indicated Flag-METTL16 vectors. Cell lysates were subjected to immunoprecipitation with Flag beads and immunoblotted with the indicated antibodies. f, HEK293T cells were transfected with empty vector or indicated Flag-METTL16 vectors. Cell lysates were incubated with Biotin-labeled control RNA or U6 snRNA and then subjected to precipitation with Streptavidin beads and immunoblotted with Flag antibody. g, HEK293T cells were transfected with empty vector or indicated Myc-MRE11 vectors. Cell lysates were incubated with Biotin-labeled control RNA or U6 snRNA and then subjected to precipitation with Streptavidin beads and immunoblotted with Myc antibody. $\mathrm{h}$ and i, Indicated concentration of purified proteins of MRE11, RAD50, NBS1, METTL16 and control RNA or U6 snRNA were incubated with 5' labeled 32P blunt h or overhang i DNA substrate in vitro. The cleavage products were shown below by autoradiography. $\mathrm{j}$ and $\mathrm{k}$, Quantification of RPA32 $\mathrm{h}$ and BrdU $\mathrm{i}$ foci in the indicated U2OS cells. At least 150 cells were analyzed. Data are presented as mean \pm SD from five independent experiments (student's $t$ test; $\left.{ }^{\star \star \star *} p<0.0001\right)$. I, HR repair efficiency in the indicated HEK293T cells. Western blot below showing expression level of METTL16. Data are presented as mean \pm SD from three independent experiments (student's t test; ${ }^{* \star *} \mathrm{p}<0.001$ ). $\mathrm{m}$, Western blots with total or chromatin-enriched extracts from indicated U2OS cells without IR or at $4 \mathrm{~h}$ after IR with $10 \mathrm{~Gy}$. Antibodies used are shown left. n, HEK293T cells were transfected with constructs encoding Flag-METTL16 WT or F187G, followed by IR with 10Gy. Cell lysates were subjected to immunoprecipitation with Flag beads and immunoblotted with the indicated antibodies. 0, HR repair efficiency in the indicated HEK293T cells. Western blot below showing expression level of METTL16. Data are presented as mean \pm SD from three independent experiments (student's $t$ test; $\star \star p<0.01, \star \star \star p<0.001$ ). $p$ and $q$, Quantification of RPA32 $p$ and BrdU $q$ foci in the indicated U2OS cells. At least 150 cells were analyzed. Data are presented as mean \pm SD from five independent experiments (student's t test; ${ }^{\star \star \star \star} p<0.0001$ ). r, Western blots with total or chromatin-enriched extracts from indicated U2OS cells without IR or at $4 \mathrm{~h}$ after IR with $10 \mathrm{~Gy}$. S, Proposed model for METTL16 attenuating MRE11 exonuclease activity thus blocking DNA end resection. 


\section{a}
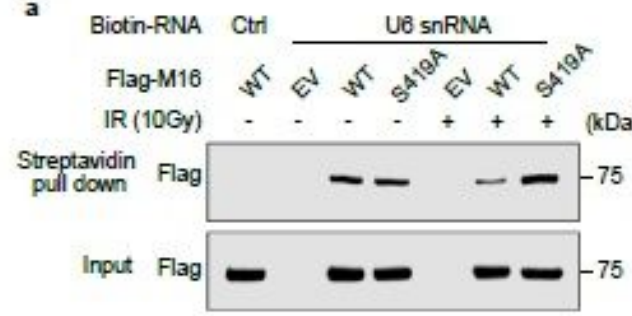

b

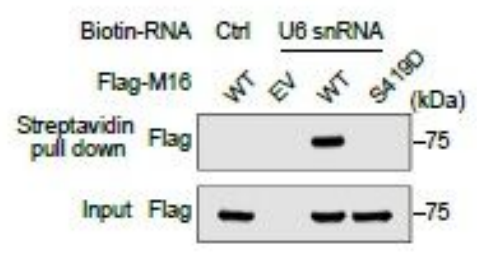

c

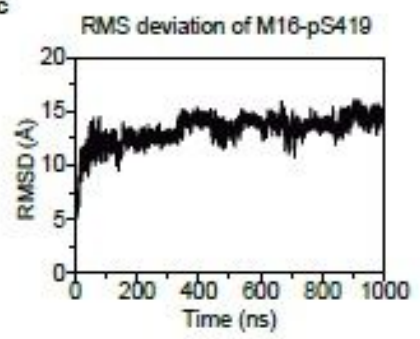

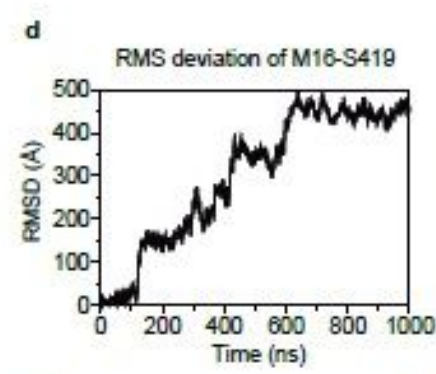
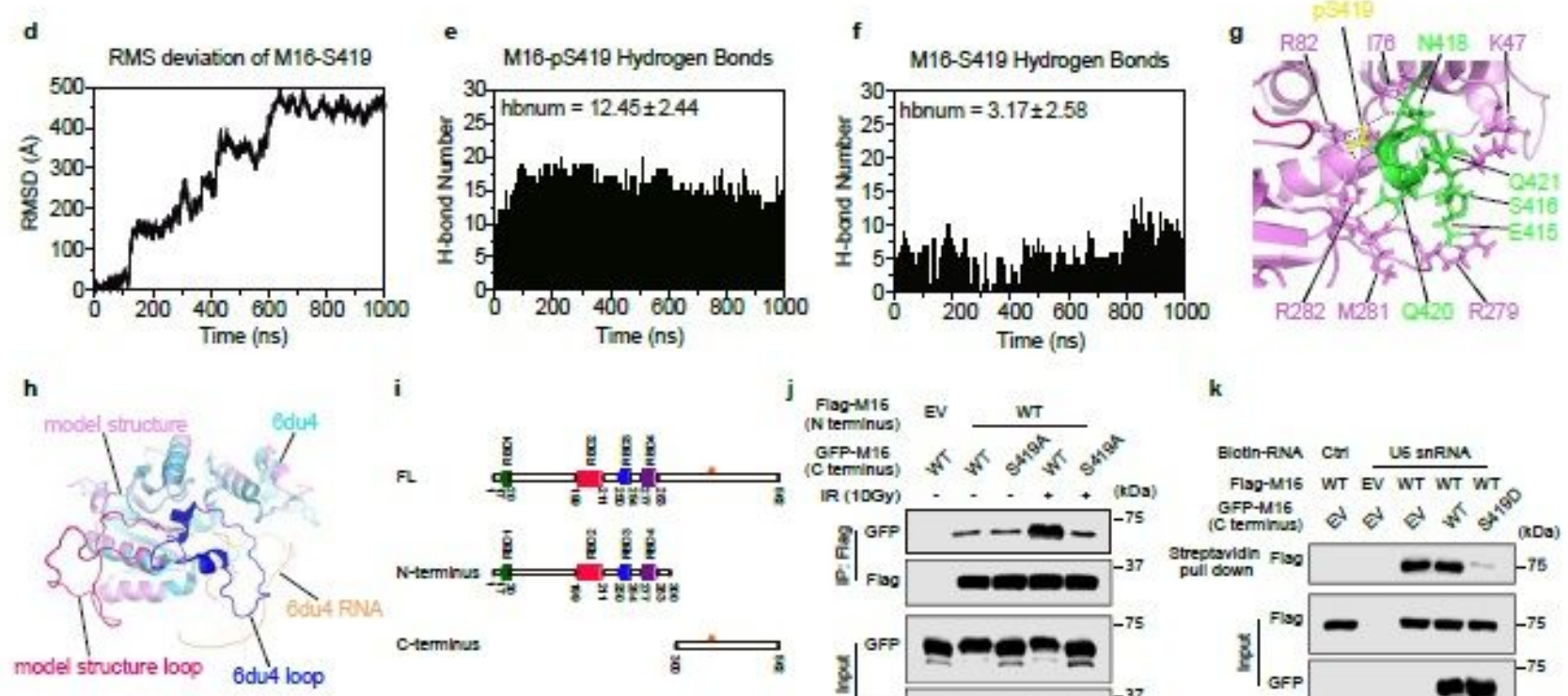

j Flag-M15 k

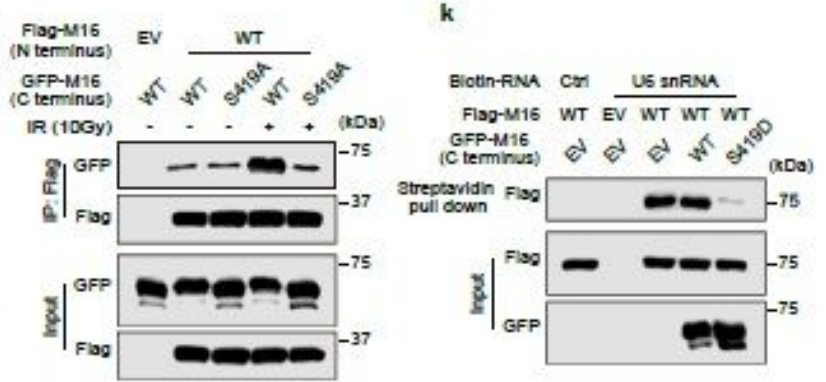

1

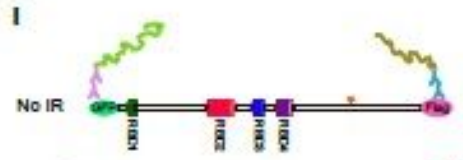

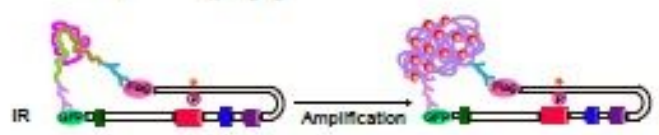

m
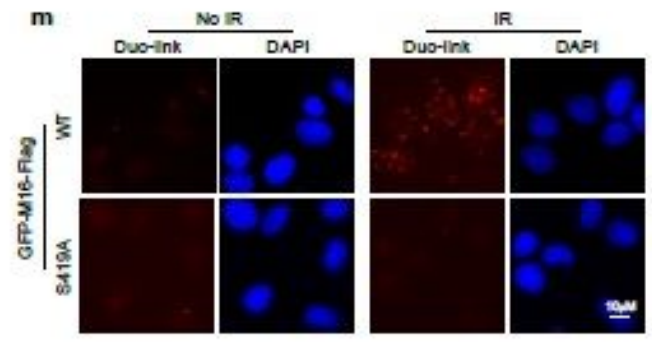

n
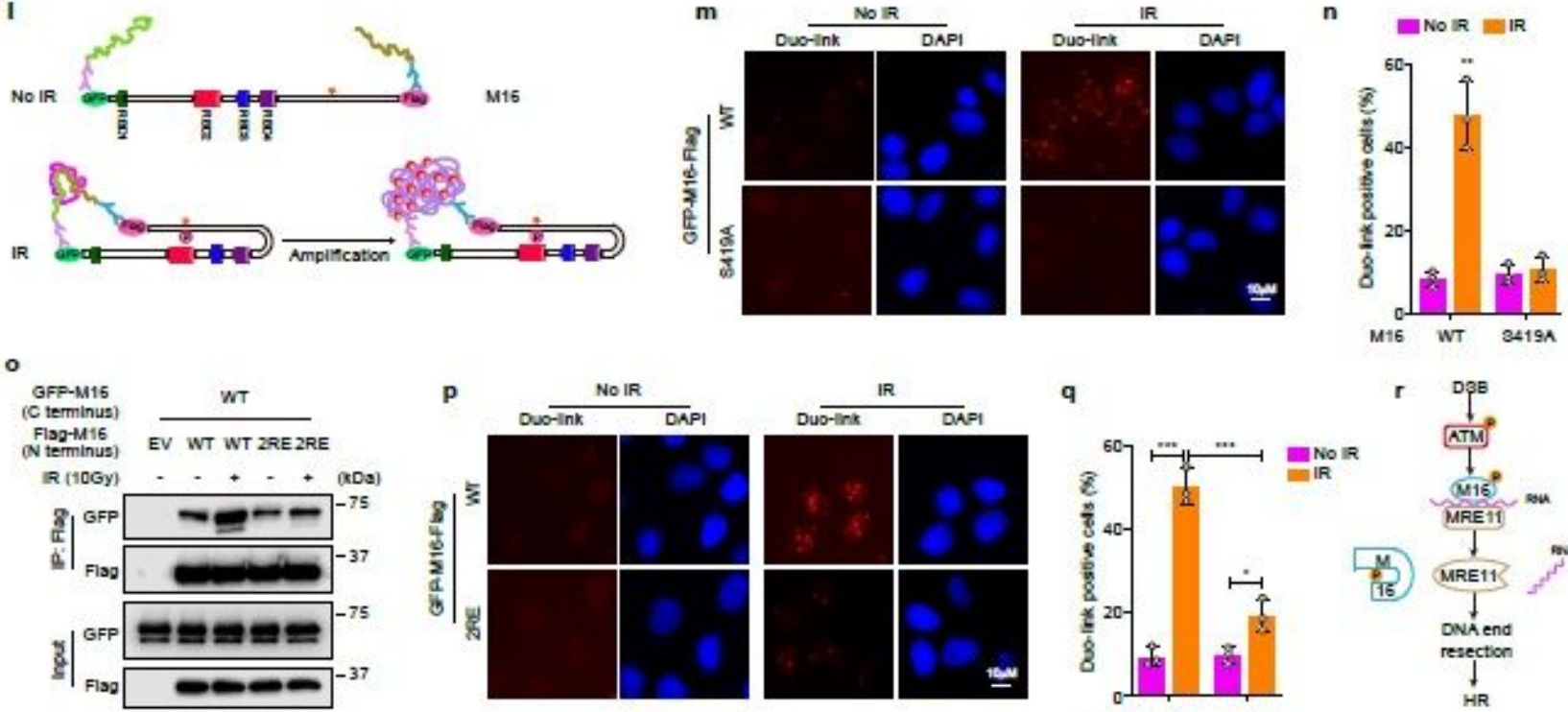
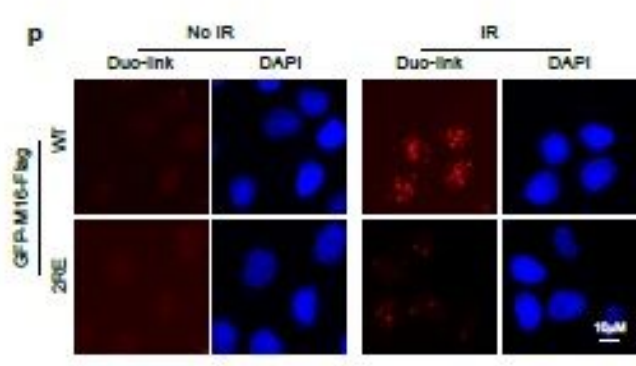
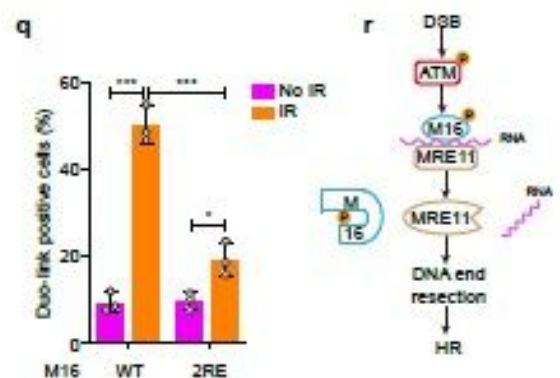

\section{Figure 5}

METTL16 phosphorylated at Ser419 induces conformational change and autoinhibited for RNA binding. a,b, HEK293T cells were transfected with empty vector or indicated Flag-METTL16 vectors with or without IR of 10 Gy. Cell lysates were incubated with Biotin-labeled control RNA or U6 snRNA and then subjected to immunoprecipitation with Streptavidin beads and immunoblotted with Flag antibody. c,d, RMS deviation of METTL16 N-terminal domain in the presence of a METTL16 (residues 413-425) 
phosphopeptide (pS419) c or unmodified peptide d. e,f, Formation of hydrogen bonds during the simulation for the METTL16 pS419 phosphopeptide e or S419 unmodified peptide $\mathrm{f}$. The average numbers of hydrogen bonds (hbnum) are indicated. g, Modeled METTL16 N-terminal domain bound to a METTL16 pS419 phosphopeptide after $1 \mathrm{~s}$ of molecular dynamics simulation. h, Molecular dynamics simulation-derived model structure of METTL16 N-terminal domain (in violet) bound to a METTL16 pS419 phosphopeptide overlaid to the X-ray structure (PDB entry 6DU4) of METTL16 N-terminal domain (in cyan) with the RNA shown in orange. The METTL16 pS419 phosphopeptide is not shown in this panel. The molecular dynamics simulation lasted $1 \mathrm{~s}$. i, Schematic structure of METTL16 truncation constructs. j, HEK293T cells were transfected with empty vector or Flag-METTL16 N terminus vector and GFP-METTL16 WT or S419A mutant C terminus vectors following IR with $10 \mathrm{~Gy}$. Cell lysates were subjected to immunoprecipitation with Flag beads and immunoblotted with the indicated antibodies. $\mathrm{k}$, HEK293T cells were transfected with empty vector or Flag-METTL16 and GFP-METTL16 C terminus vector. Cell lysates were incubated with Biotin-labeled control RNA or U6 snRNA and then subjected to immunoprecipitation with Streptavidin beads and immunoblotted with Flag or GFP antibodies. I, A diagram of Duo-link in situ assay designed for following experiments. $m, n$, Representative images $m$ and quantification $n$ of Duo-link in situ in U2OS cells expressing WT or S419A mutation GFP-METTL16-Flag with or without IR. At least 150 cells were analyzed. Data are presented as mean \pm SD from three independent experiments (student's t test; ${ }^{\star \star} p<0.01$ ). Scale bar is indicated. o HEK293T cells were transfected with GFP-METTL16 C terminus vector and Flag-METTL16 WT or 2RE mutant N terminus vectors following IR with $10 \mathrm{~Gy}$. Cell lysates were subjected to immunoprecipitation with Flag beads and immunoblotted with the indicated antibodies. p,q, Representative images $p$ and quantification q of Duolink in situ in U2OS cells expressing WT or 2RE mutation GFP-METTL16-Flag with or without IR. At least 150 cells were analyzed for each datum point. Data are presented as mean \pm SD from three independent experiments (student's $t$ test; ${ }^{\star} p<0.05,{ }^{\star \star \star} p<<0.001$ ). Scale bar is indicated. $r$, Proposed model for DNA damage induces METTL16 conformational change and autoinhibited for RNA binding. 

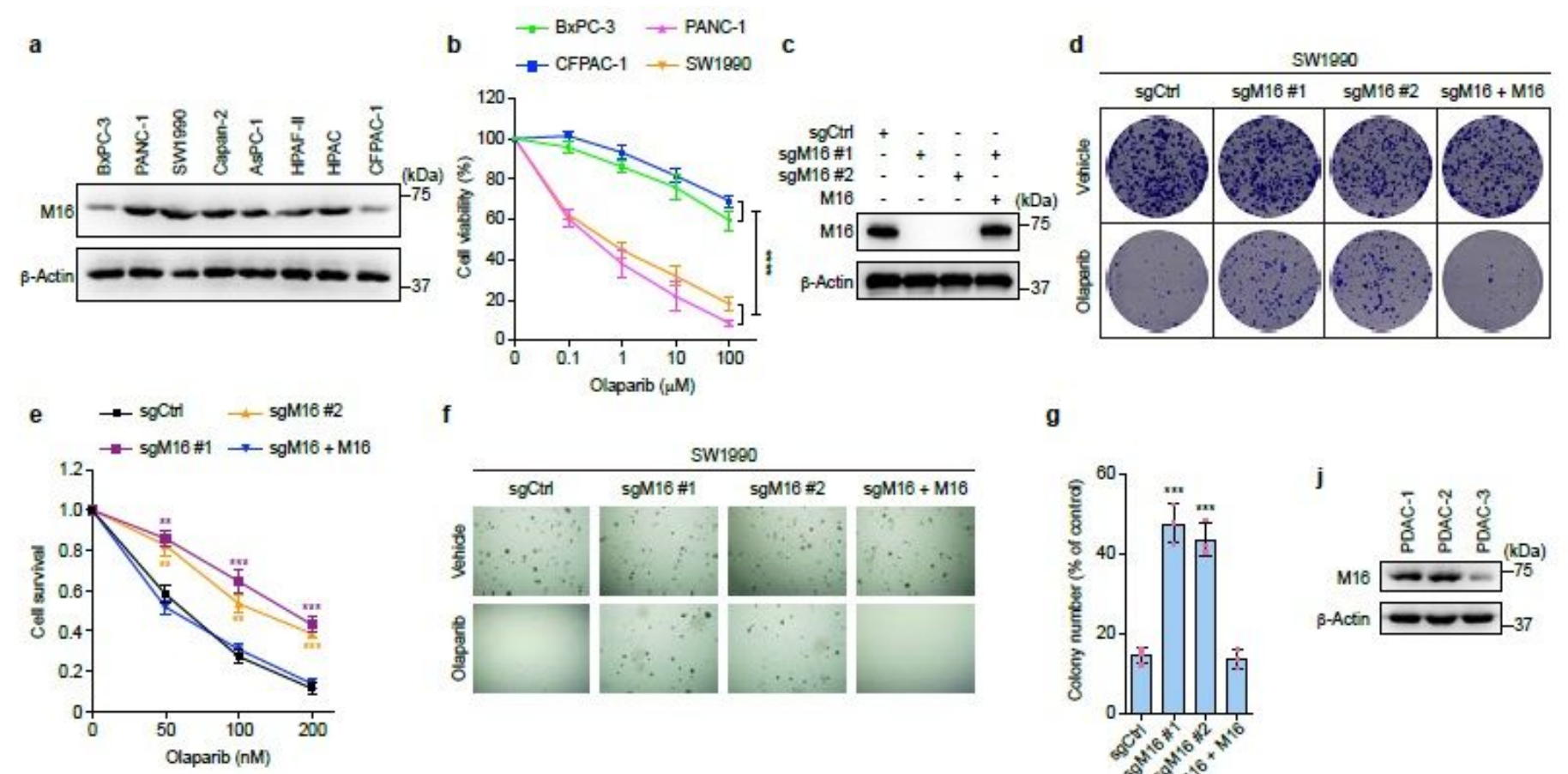

f

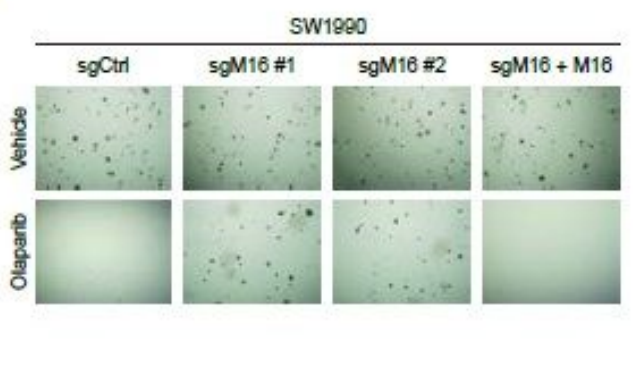

g

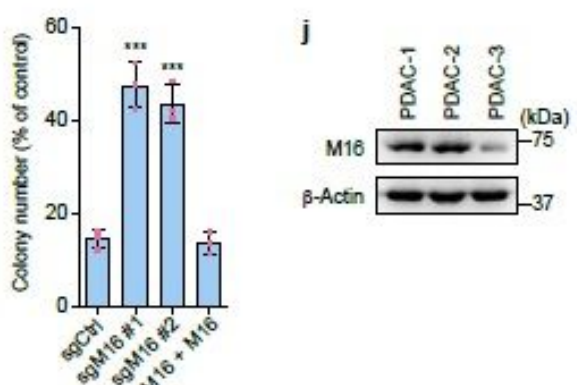

h
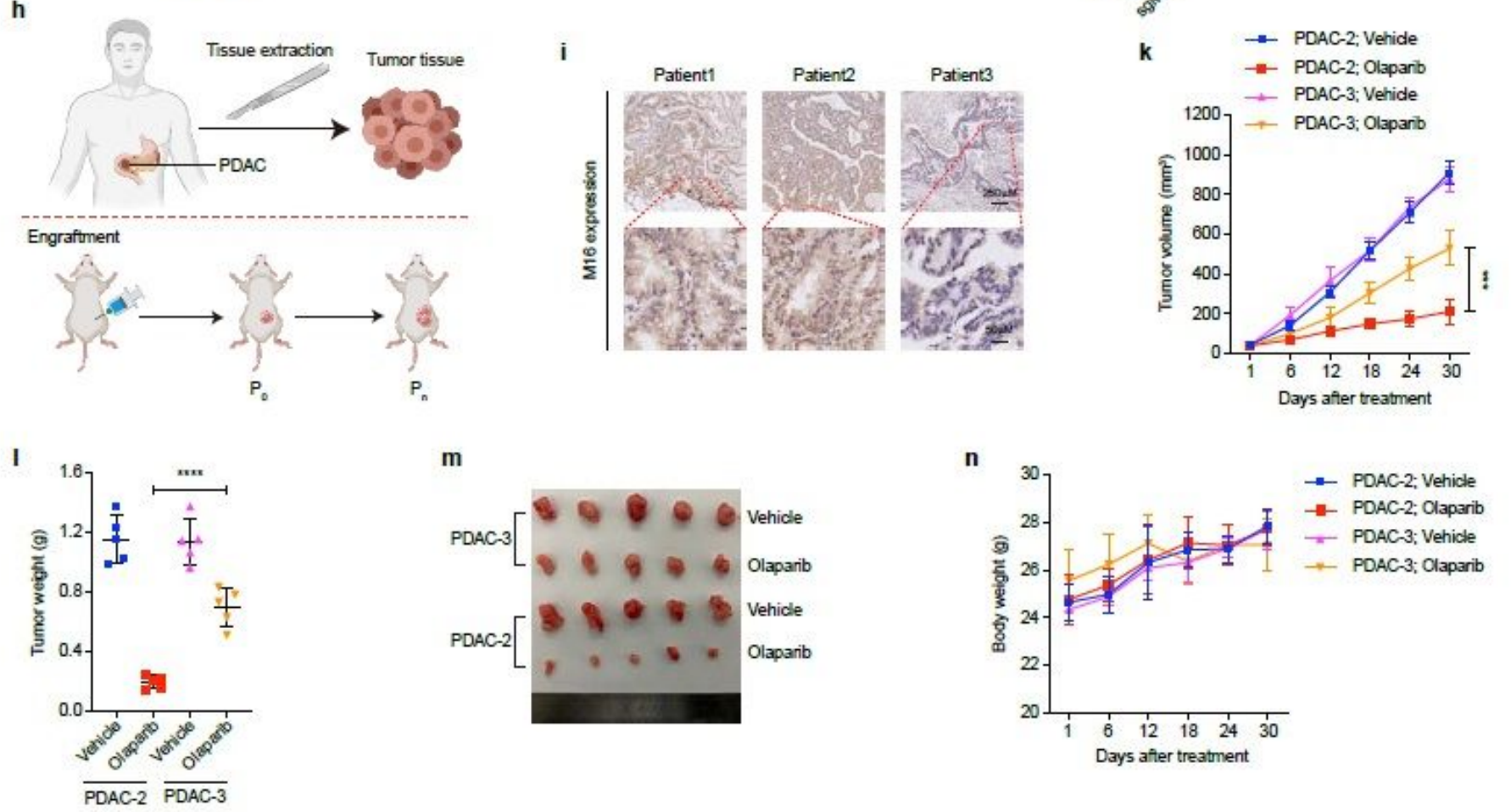

\section{Figure 6}

High METTL16 expression is correlated with increased sensitivity to PARPi. a, Immunoblot of METTL16 in different PDAC cell lines. b, MTS assay to evaluate the proliferation of indicated four PDAC cell lines after exposure to olaparib for $72 \mathrm{~h}$. Data are presented as mean \pm SD from three independent experiments (two-way ANOVA; ****p < 0.0001). c, Immunoblot of METTL16 in METTL16 KO SW1990 cells expressing WT METTL16. b-Actin was used as a loading control. d,e, Representative images $d$ and quantification e of 2-D colony formation assay in the indicated SW1990 cells treated with olaparib. Data are presented as mean \pm SD from three independent experiments (two-way ANOVA; $* * p<0.01, * \star \star * p<0.0001$ ). $f, g$, 
Representative images $f$ and quantification $g$ of soft agar colony formation in the indicated SW1990 cells treated with olaparib. Data are presented as mean \pm SD from three independent experiments (student's $t$ test; $\left.{ }^{\star \star *} p<0.001\right)$. $h$, Schematic model for generating patient-derived tumor xenograft of PDAC. i, Representative IHC micrographs showing METTL16 expression in three PDAC patients. Scale bars are indicated. j, Immunoblot of METTL16 in three PDAC PDX tissues. k, Growth curves of the PDAC-2 and PDAC-3 PDX models treated with vehicle or olaparib. Data are presented as mean \pm SD (two-way ANOVA; $\star \star \star p<0.001)$. I, Quantification of the weight of the tumors in different groups of the PDX models. Data are presented as mean \pm SD (student's $t$ test; ${ }^{\star \star \star \star} \mathrm{p}<0.0001$ ). $\mathrm{m}$, Photograph of the tumors harvested at the end of the experiment with the PDX models. $n$, Body weight of the mice in different groups of the PDX models. 

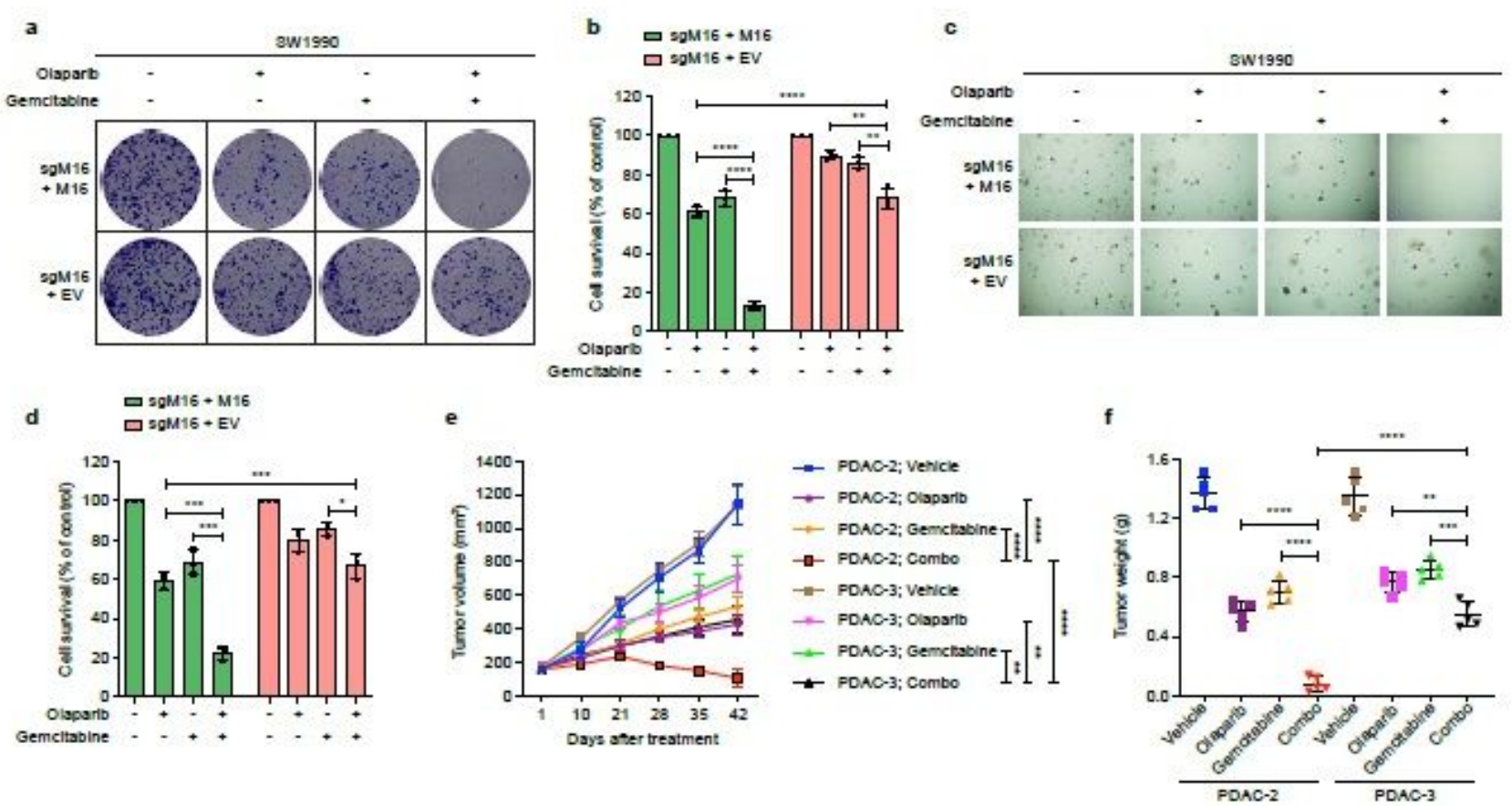

g

h
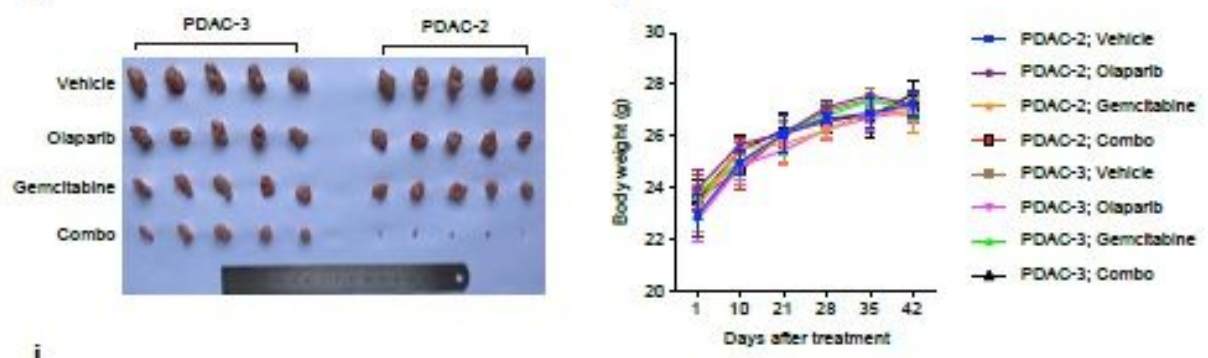

i

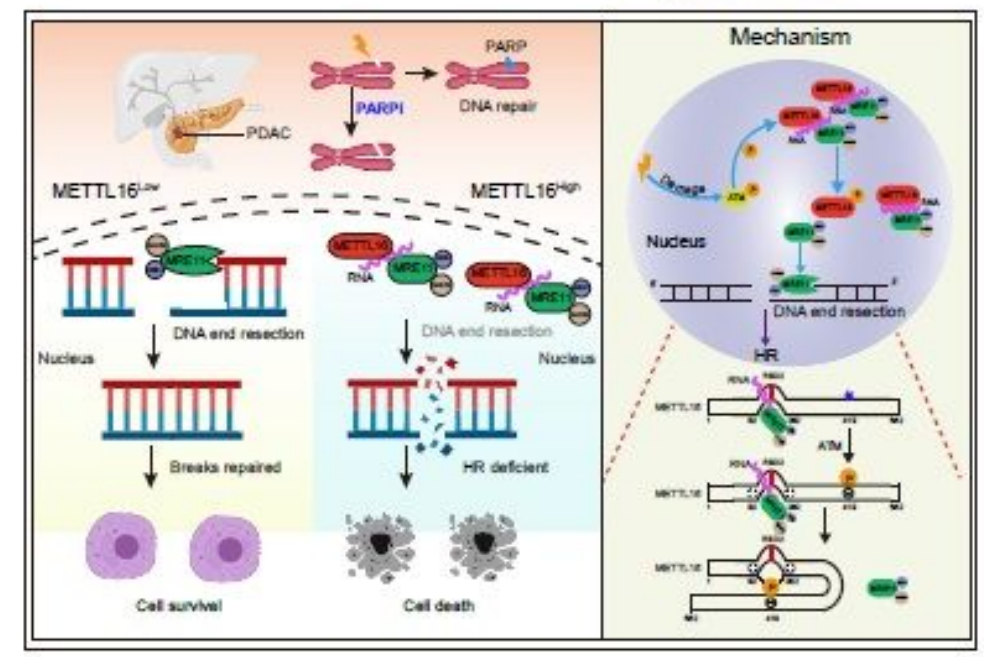

\section{Figure 7}

PARPi and Gemcitabine in combination effectively kill tumor cell-expression METTL16. a,b, Representative images a and quantification b of 2-D colony formation assay in the indicated SW1990 cells treated with olaparib or gemcitabine. Data are presented as mean \pm SD from three independent experiments (two-way ANOVA; $\star \star x p<0.01, \star \star \star \star p<<0.0001$ ). c,d, Representative images $c$ and quantification d of soft agar colony formation in the indicated SW1990 cells treated with olaparib or gemcitabine. Data 
are presented as mean \pm SD from three independent experiments (student's $t$ test; ${ }^{*} p<0.05$, ${ }^{\star \star \star} p<0.001$ ). e, Growth curves of the PDAC-2 and PDAC-3 PDX models treated with vehicle, olaparib, gemcitabine or combo. Data are presented as mean \pm SD (two-way ANOVA; $* * p<0.01, * \star * * p<0.0001$ ). $f$, Quantification of the weight of the tumors in different groups of the PDX models. Data are presented as mean \pm SD (student's t test; ${ }^{* \star} p<0.01,{ }^{\star \star \star} p<0.001,{ }^{\star \star \star \star} p<0.0001$ ). g, Photograph of the tumors harvested at the end of the experiment with the PDX models. $h$, Body weight of the mice in different groups of the PDX models. i, A schematic model to show that METTL16 expressing in PDAC confers synthetic lethality to PARP inhibition by attenuating MRE11-mediated DNA end resection.

\section{Supplementary Files}

This is a list of supplementary files associated with this preprint. Click to download.

- ExtendedDataFigures.pdf 